$s$

ENGINEERING CHANGE NOTICE

Page 1 of Z

1. ECN 654680

Proj.

ECN

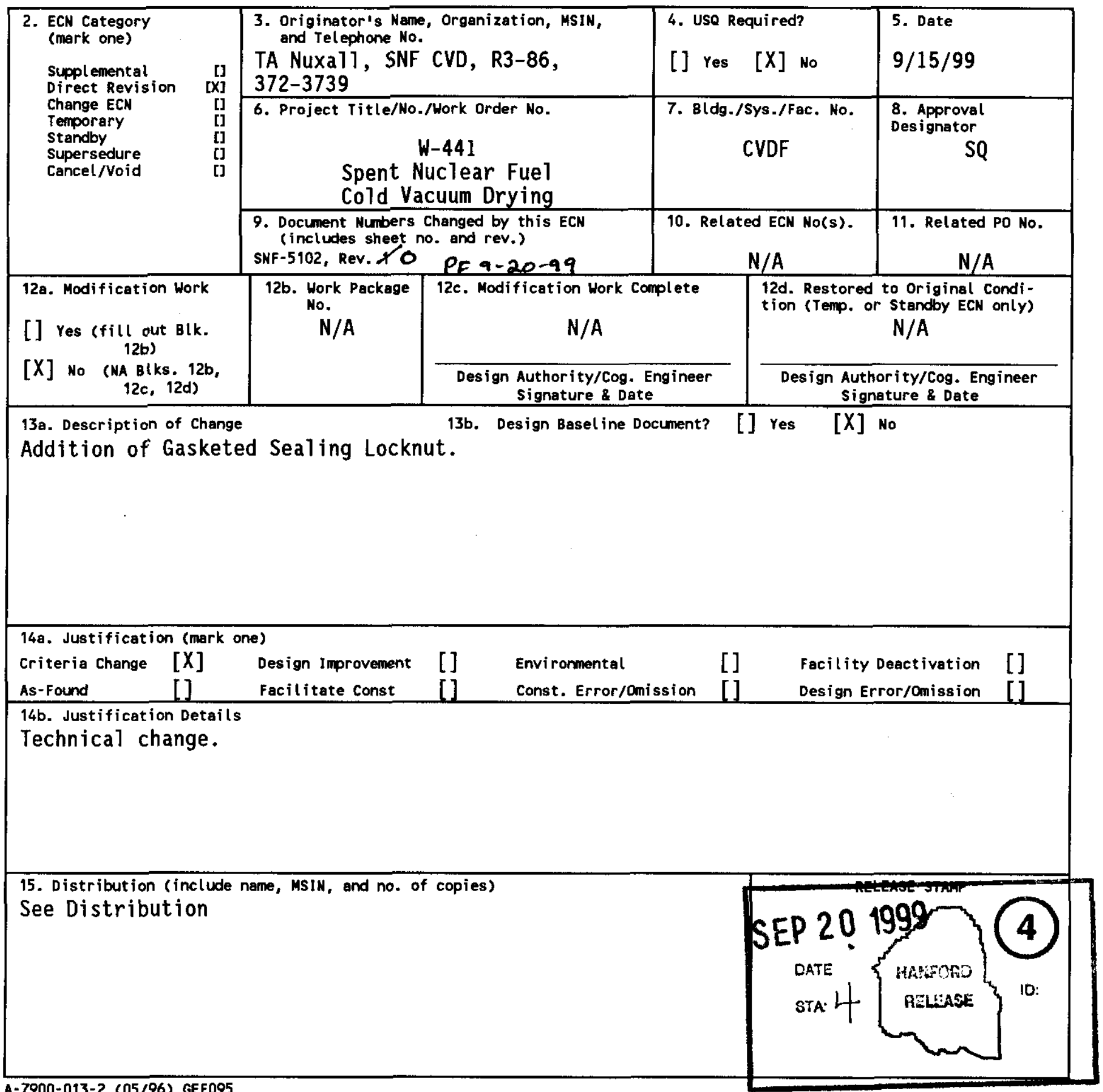




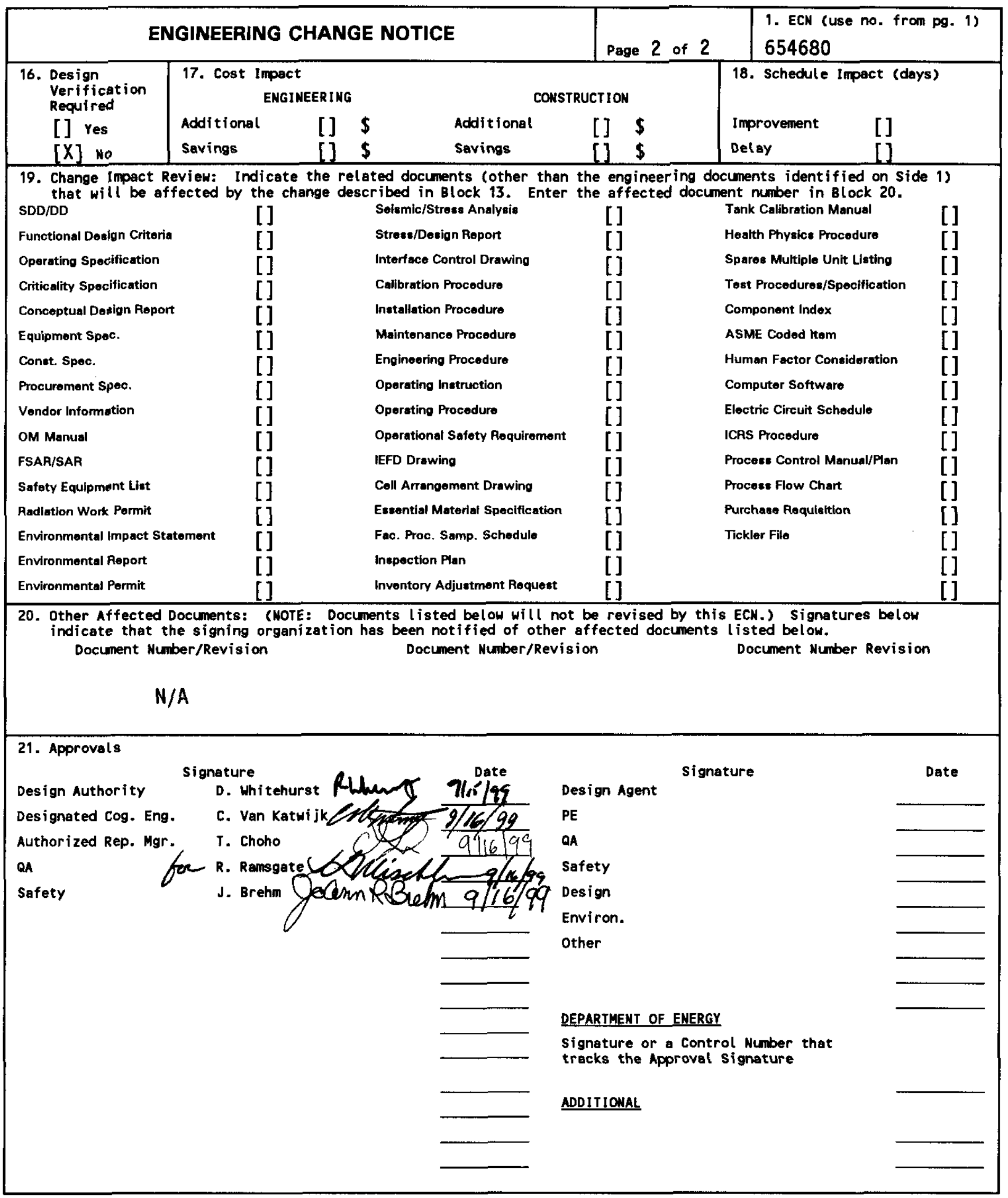




\section{CONDUIT FASTENERS}

Carl Van Katwijk

Numatec Hanford, Richland, WA 99352

U.S. Department of Energy Contract DE-AC06-96RL13200

EDT/ECN: 654680

UC: 620

Org Code: $2 \mathrm{G} 300$

Charge Code: 105559/A000

B\&R Code: 39EW40400

Total Pages: 3635

$$
\text { PF } 9-20-99
$$

Key Words: W-441, Cold Vacuum Drying Facility (CVD), CGI, Conduit Fasteners

Abstract: W-441, Cold Vacuum Drying Facility (CVD), CGI, Conduit Fasteners

TRADEMARK DISCLAIMER. Reference herein to any specific commercial product, process, or service by trade name, trademark, manufacturer, or otherwise, does not necessarily constitute or imply its endorsement, recommendation, or favoring by the United States Government or any agency thereof or its contractors or subcontractors.

Printed in the United States of America. To obtain copies of this document, contact: Docum znt Control services, P.O. Box 950, Mailstop H6-08, Richland WA 99352, Phone (509) 372-2420; Fax (509) 376-4989.

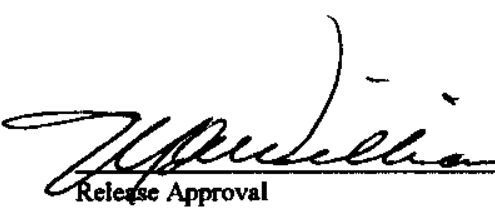

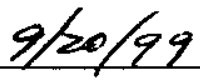

(1)

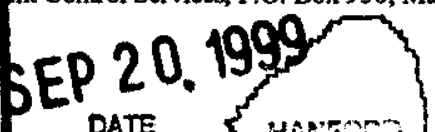

DATE
Hân'AR

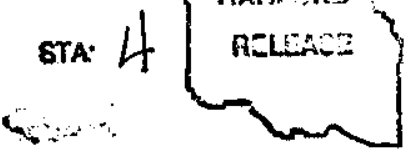




\section{RECORD OF REVISION}

(2) Title

SNF-5102, CONDUIT FASTENERS

Change Control Record

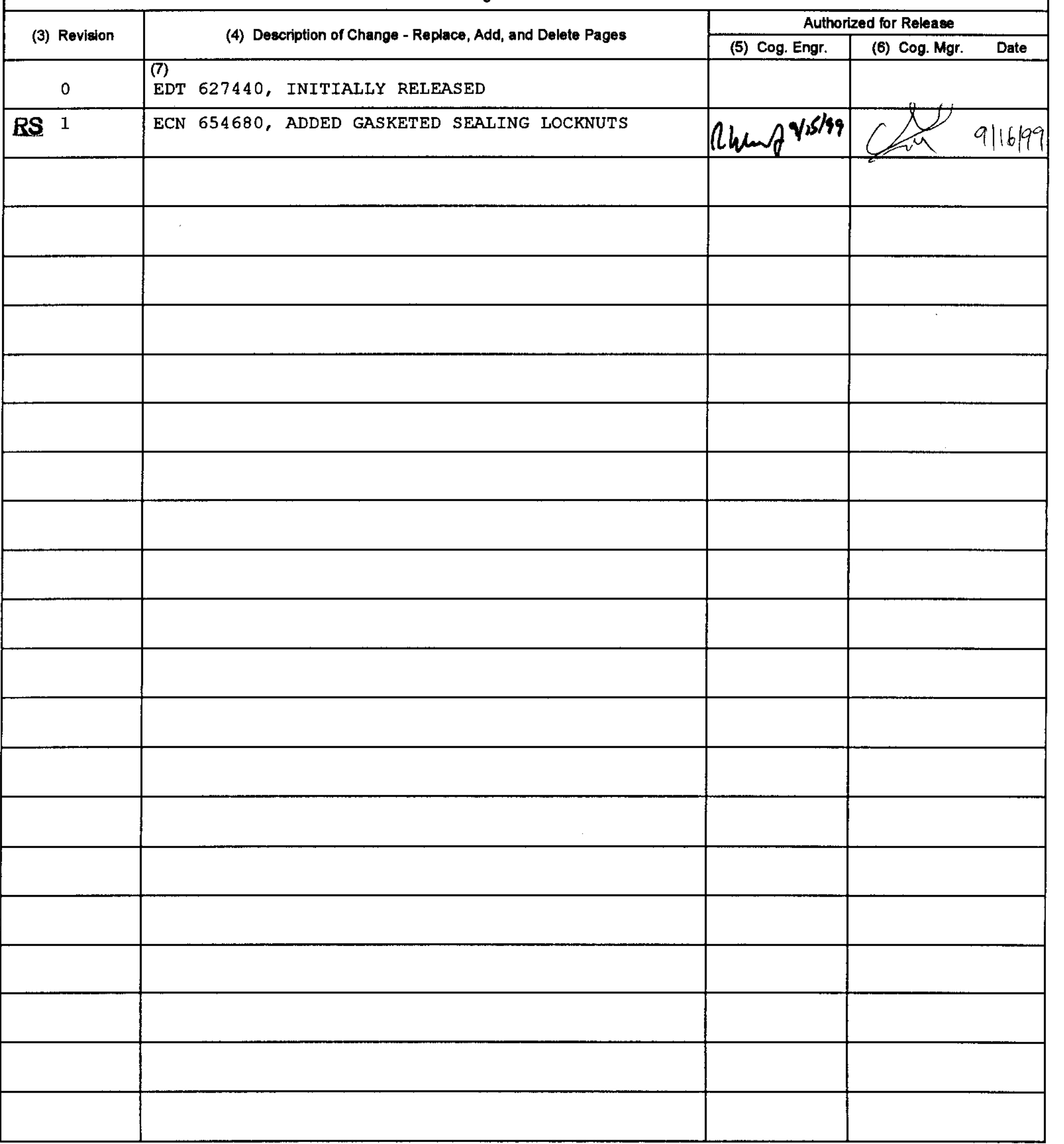




\begin{tabular}{|l|l|}
\hline Commercial Grade Item Upgrade Dedication Form & SNF-5102 Rev. 1 \\
\hline $\begin{array}{l}\text { ECN No. NA } \\
\text { Title: CONDUIT FASTENERS }\end{array}$ & Page 1 of 33 \\
\hline
\end{tabular}

\begin{tabular}{|c|c|c|c|}
\hline \multicolumn{4}{|c|}{ Section 1 Part Intomation } \\
\hline Item No.: NA & \multicolumn{2}{|l|}{ Manufacturer: } & Supplier: \\
\hline \multicolumn{2}{|l|}{ Mfg. Part/Model No.: } & \multicolumn{2}{|l|}{ Supplier's PIN: } \\
\hline \multicolumn{4}{|l|}{ Part Description: } \\
\hline \multicolumn{4}{|l|}{ End Use Description: } \\
\hline \multicolumn{4}{|c|}{ Section 2 a Component liformation } \\
\hline $\begin{array}{l}\text { Equipment No.: See } \\
\text { attached Section } 9\end{array}$ & $\begin{array}{l}\text { Specification No.: W-441- } \\
\text { C1, Section } 16110\end{array}$ & $\begin{array}{l}\text { Manufacturer: } \\
\text { ABC Fasteners; } \\
\text { PowerStrut; Fastenal }\end{array}$ & Past P.O. No.: NA \\
\hline $\begin{array}{l}\text { Procurement and/or } \\
\text { Model No.: See attached } \\
\text { Section } 9\end{array}$ & $\begin{array}{l}\text { Equipment Supplier (if differer } \\
\text { American Electric: } \\
\text { George A, Grant, Inc fo }\end{array}$ & $\begin{array}{l}\text { from manufacturer): } \\
\text { Fastenal }\end{array}$ & $\begin{array}{l}\text { Equip. Supplier's Part No.: } \\
\text { NA }\end{array}$ \\
\hline \multicolumn{4}{|c|}{ Component Description: Fasteners for Safety Class Wiring Conduit. } \\
\hline \multicolumn{4}{|c|}{ Section 26 Commercial Availability of the ltem } \\
\hline \multicolumn{4}{|c|}{$\begin{array}{l}\text { 1. Is the Item available from a catalogue of a qualified NQA1 supplier? (coordinate with project CGI interface Engineer or BTR) } \\
\text { [ ] YES (go to \#2 below) } \\
\text { [X] NO (go to procedure step 6.3.2, proceed to dedicate Item.) } \\
\text { If not available from a qualified NQA1 supplier, is it avaliable from an ISO } 9000 \text { supplier? (coordinate with project CGI interface } \\
\text { Engineer or BTR) }\end{array}$} \\
\hline \multicolumn{4}{|c|}{$\begin{array}{l}\text { 2. List of Candidate qualified suppliers or ISO } 9000 \text { suppliers } \\
\text { company name \& type }\end{array}$} \\
\hline \multicolumn{4}{|l|}{ NA } \\
\hline \multicolumn{4}{|c|}{$\begin{array}{l}\text { 3. Recommended Procurement Strategy (coordinate with project CGl interface Engineer or BTR): } \\
\text { NA }\end{array}$} \\
\hline \multicolumn{4}{|c|}{ Soction 20 CGI Dotemination } \\
\hline \multicolumn{4}{|c|}{$\begin{array}{l}\text { 1. Question \#1: Is the Item subject to design or specification requirements that are unique to nuclear facilities or activities? } \\
\text { [ ] YES (the Item is not commercial grade) } \\
\text { [X] NO (continue) }\end{array}$} \\
\hline \multicolumn{4}{|c|}{$\begin{array}{l}\text { 2. Question \#2: Is the Item used in applications other than nuclear facilities or activities? } \\
\text { [ ] NO (the item is not commercial grade) } \\
{[X] \text { YES (continue) }}\end{array}$} \\
\hline \multicolumn{4}{|c|}{$\begin{array}{l}\text { 3. Question \#3: Is the Item ordered from manufacturer/supplier o } \\
\text { information (e.g. manufacturer's catalog)? } \\
\text { [ ] NO (the Item is not commercial grade) } \\
\text { [X] YES (continue) }\end{array}$} \\
\hline
\end{tabular}




\begin{tabular}{|l|l|}
\hline Commercial Grade Item Upgrade Dedication Form & SNF-5102 Rev. 1 \\
\hline $\begin{array}{l}\text { ECN No. NA CGI No. CGI-SNF-D-93-2-C1-056 } \\
\text { Title: CONDUIT FASTENERS }\end{array}$ & Page 2 of 33 \\
\hline
\end{tabular}

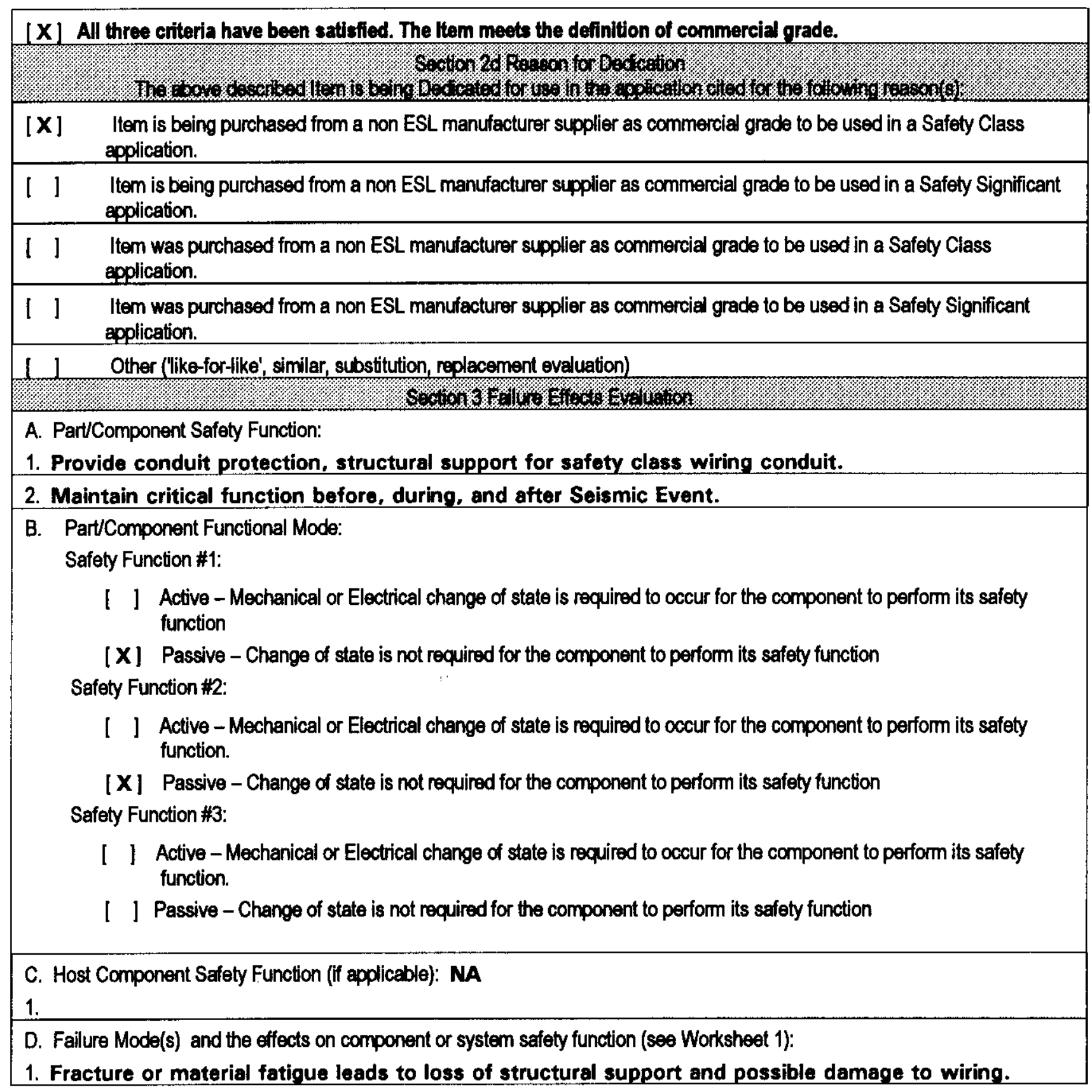




\begin{tabular}{|l|l|}
\hline Commercial Grade Item Upgrade Dedication Form & SNF-5102 Rev. 1 \\
\hline $\begin{array}{l}\text { ECN No. NA CGI No. CGI-SNF-D-93-2-C1-056 } \\
\text { Title: CONDUIT FASTENERS }\end{array}$ & Page 3 of 33 \\
\hline
\end{tabular}

\begin{tabular}{|c|c|c|c|}
\hline \multicolumn{2}{|l|}{$\begin{array}{l}\text { Environmental Qualification Required: } \\
\text { Yes [ ] } \\
\text { No }[\mathbf{X}] \\
\text { Environmental Condition B }\end{array}$} & \multicolumn{2}{|c|}{$\begin{array}{l}\text { If yes: Environmental Qualification Requirements } \\
\text { Limiting Environmental Conditions: } \\
\text { Required Safety Functions: }\end{array}$} \\
\hline \multirow{3}{*}{\multicolumn{2}{|c|}{$\begin{array}{l}\text { Natural Phenomena Hazard (NPH) Design Required: } \\
\text { Yes }[\mathbf{X}] \\
\text { No }\end{array}$}} & \multicolumn{2}{|c|}{ If yes: NPH Design Requirements } \\
\hline & & \multicolumn{2}{|c|}{ Performance Category: PC-3 } \\
\hline & & \multicolumn{2}{|c|}{ NPH Design Req'ts.: Seismic Condition C } \\
\hline HNF-PRO-097, Rev. 0 & & \multicolumn{2}{|c|}{$\begin{array}{l}\text { Required Safety Functions: Provide protection and } \\
\text { support for safety class wiring conduit. Maintain } \\
\text { critical function before, during, and after Seismic } \\
\text { Event. }\end{array}$} \\
\hline \multicolumn{4}{|c|}{ 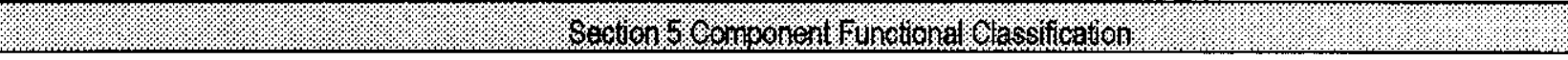 } \\
\hline \multicolumn{4}{|c|}{$\begin{array}{l}\text { [X] Safety Class (SC) I ] General Service (GS) [ ] Safety } \\
\text { If part/component classification is different from host component/system, document basis. NA }\end{array}$} \\
\hline \multicolumn{4}{|c|}{ Section 6 (Resenved) } \\
\hline \multicolumn{4}{|c|}{ Sectoon 7 (Resenod) } \\
\hline \multicolumn{4}{|c|}{ Section 8 Rererences (for Functional Classification) } \\
\hline $\begin{array}{l}\text { National Codes/Standards: IEEE } \\
628\end{array}$ & $\begin{array}{l}\text { Safety Analy } \\
\text { HNF-SD-S } \\
\text { Rev. 4A }\end{array}$ & (SAR): & $\begin{array}{l}\text { Drawings: HNF-SD-SNF-SEL-002, } \\
\text { Rev. 6A } \\
\text { Dwg. H-1-82303 }\end{array}$ \\
\hline \multicolumn{4}{|c|}{$\begin{array}{l}\text { Vendor Manual/Manufacturer/Supplier Information: } \\
\text { Fender Washers, Lock Washers, Flat Washers, Hex Head Bolts - ABC Fasteners Page 2-1; } \\
\text { PS-146 Continuous Threaded Rod/Clamping Nut with spring - Power Strut Pages } 40,42 \\
\text { Phillips Drive Machine Screws - Fastenal Catalog, Pages } 76 \text { and } 77 \\
\text { Nylon Insert Lock Nuts, Fastenal Catalog, Page } 104 \\
\text { Hex Full Nuts Fastenal Catalog, Page } 101 \\
\text { SAE Flat Washers, Fastenal Catalog, Page } 115 \\
\text { Medium Split Lock Washers, Fastenal Catalog, Page } 116\end{array}$} \\
\hline Other: & & & \\
\hline
\end{tabular}




\begin{tabular}{|l|l|}
\hline Commercial Grade Item Upgrade Dedication Form & SNF-5102 Rev. 1 \\
\hline $\begin{array}{l}\text { ECN No. NA CGI No. CGI-SNF-D-93-2-C1-056 } \\
\text { Title: CONDUIT FASTENERS }\end{array}$ & Page 4 of 33 \\
\hline
\end{tabular}

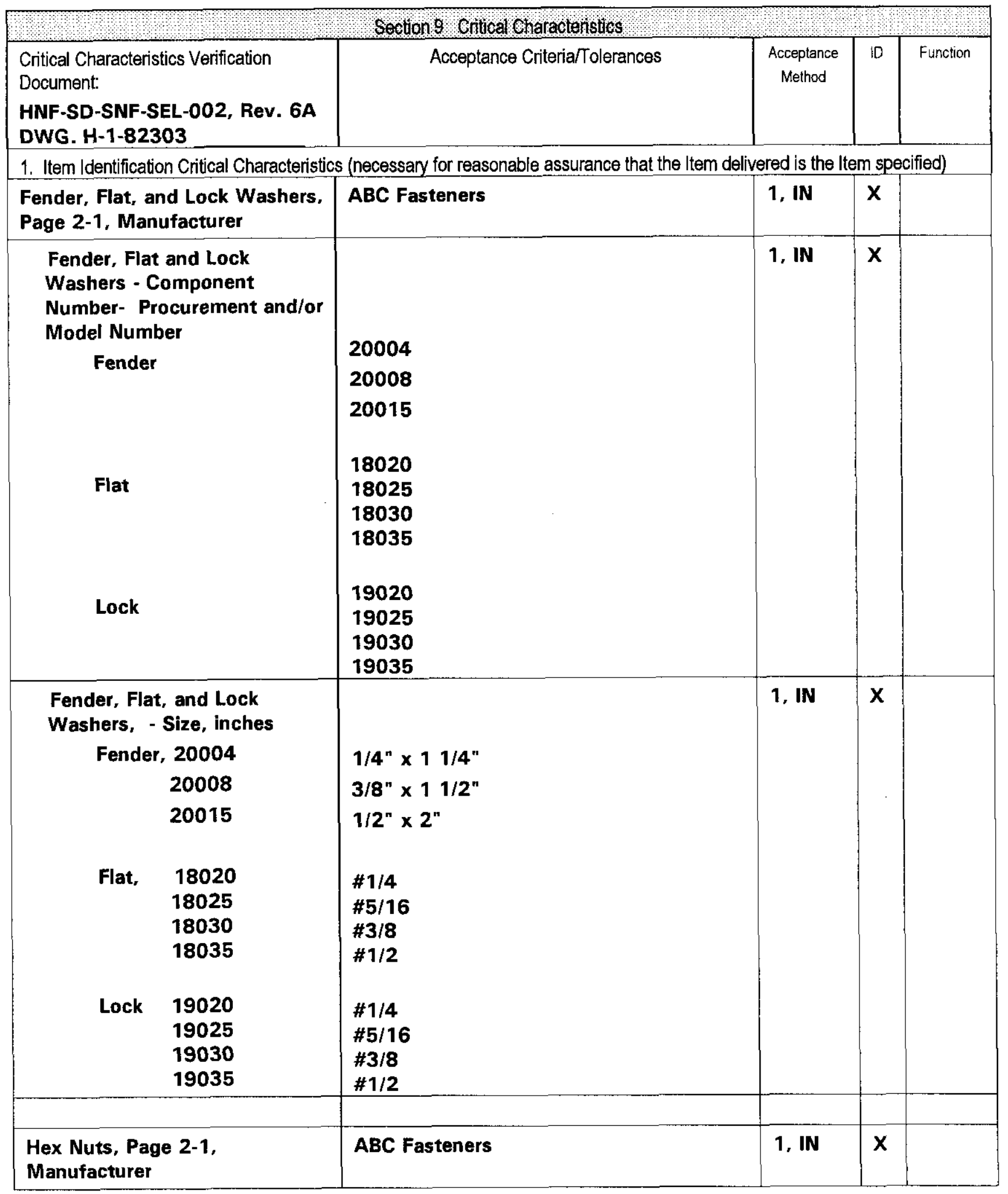




\begin{tabular}{|l|l|}
\hline Commercial Grade Item Upgrade Dedication Form & sNF-5102 Rev. 1 \\
\hline $\begin{array}{l}\text { ECN No. NA CGI No. CGI-SNF-D-93-2-C1-056 } \\
\text { Title: CONDUIT FASTENERS }\end{array}$ & Page 5 of 33 \\
\hline
\end{tabular}

\begin{tabular}{|c|c|c|c|}
\hline $\begin{array}{l}\text { Hex Nuts, - Component } \\
\text { Number- Procurement and/or } \\
\text { Model Number }\end{array}$ & $\begin{array}{l}17025 \\
17030 \\
17035 \\
17040 \\
\end{array}$ & 1, IN & $\mathbf{x}$ \\
\hline $\begin{array}{l}\text { Hex Nuts, - Size/Thread, inch } \\
17025 \\
17030 \\
17035 \\
17040\end{array}$ & $\begin{array}{l}1 / 4-20 \\
5 / 16-18 \\
3 / 8-16 \\
1 / 2-13\end{array}$ & 1, IN & $\mathbf{x}$ \\
\hline Hex Head Bolts, Manufacturer & ABC Fasteners & 1, IN & $\underline{x}$ \\
\hline $\begin{array}{l}\text { Hex Head Bolts, - } \\
\text { Component Number- } \\
\text { Procurement and/or Model } \\
\text { Number } \\
\quad 1 / 4 \text { inch } \\
5 / 16 \text { inch } \\
\quad 3 / 8 \text { inch }\end{array}$ & $\begin{array}{l}38110 \\
38111 \\
38112 \\
38113 \\
\mathbf{3 8 1 1 4} \\
38115 \\
38126 \\
38127 \\
38128 \\
38141 \\
38142 \\
38143 \\
38144 \\
\end{array}$ & 1, IN & $\bar{x}$ \\
\hline $\begin{array}{l}\text { Hex Head Bolts, - Size - } \\
\text { Thread x Nominal Length, } \\
\text { inch } 38110 \\
38111 \\
38112 \\
38113 \\
38114 \\
38115 \\
\\
38126 \\
38127 \\
38128 \\
\\
38141 \\
38142 \\
38143 \\
38144\end{array}$ & 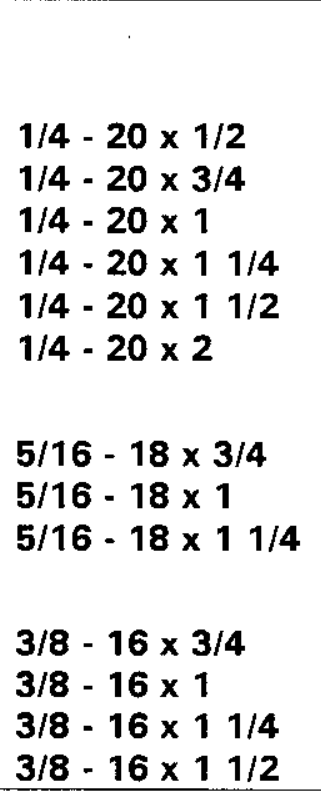 & 1, IN & $\mathbf{x}$ \\
\hline
\end{tabular}




\begin{tabular}{|l|l|}
\hline Commercial Grade Item Upgrade Dedication Form & SNF-5102 Rev. 1 \\
\hline $\begin{array}{l}\text { ECN No. NA CGI No. CGI-SNF-D-93-2-C1-056 } \\
\text { Title: CONDUIT FASTENERS }\end{array}$ & Page 6 of 33 \\
\hline
\end{tabular}

\begin{tabular}{|c|c|c|c|}
\hline $\begin{array}{l}\text { Fasteners, Pages } 40 \text { and } 42 \\
\text { Manufacturer } \\
\text { PS } 146 \text { Continuous } \\
\text { Threaded Rod - Size } \\
\text { PS RS Clamping Nut with } \\
\text { Regular Spring. } \\
\text { PS RS - Size } x \text { Thread }\end{array}$ & $\begin{array}{l}\text { Powerstrut } \\
\text { Nominal 3/8" } \\
\text { Nominal } 1 / 2^{\prime \prime} \\
\text { Nominal } 5 / 8^{\prime \prime} \\
\text { Nominal } 3 / 4^{\prime \prime} \\
1 / 4^{\prime \prime} \times 20 \\
3 / 8^{\prime \prime} \times 16 \\
1 / 2^{\prime \prime} \times 13 \\
5 / 8^{\prime \prime} \times 11 \\
3 / 4^{n} \times 10 \\
7 / 8^{n} \times 9\end{array}$ & 1 , IN & $x$ \\
\hline $\begin{array}{l}\text { Manufacturer, Pan Head Phillips } \\
\text { Drive Machine Screws - All - } \\
\text { Pages } 76 \text { and } 77\end{array}$ & Fastenal & 1, IN & $\mathbf{x}$ \\
\hline $\begin{array}{l}\text { Pan Head Phillips Drive } \\
\text { Machine Screws - Size } \\
\text { - Thread, Part Number } \\
\text { / Nominal Length, } \\
\text { Inches }\end{array}$ & 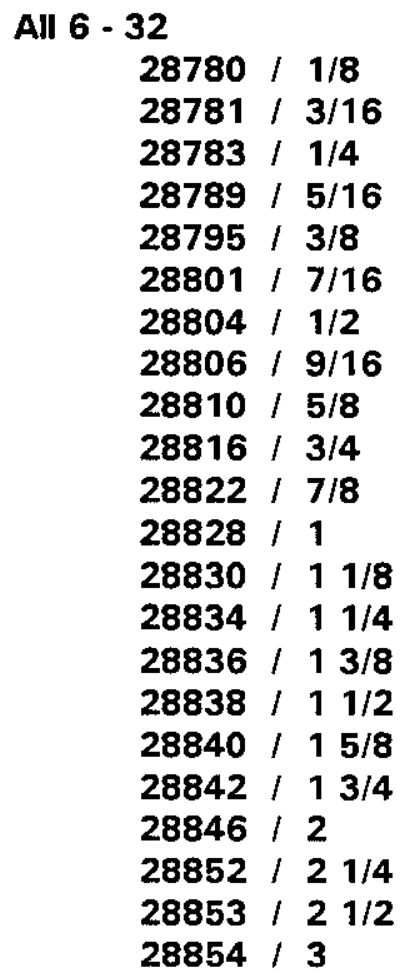 & 1, IN & $x$ \\
\hline
\end{tabular}




\begin{tabular}{|l|l|}
\hline Commercial Grade Item Upgrade Dedication Form & SNF-5102 Rev. 1 \\
\hline $\begin{array}{l}\text { ECN No. NA CGI No. CGI-SNF-D-93-2-C1-056 } \\
\text { Title: CONDUIT FASTENERS }\end{array}$ & Page 7 of 33 \\
\hline
\end{tabular}

\begin{tabular}{|c|c|c|c|}
\hline $\begin{array}{l}\text { Pan Head Phillips Drive } \\
\text { Machine Screws - Size } \\
\text { - Thread, Part Number } \\
\text { / Nominal Length, } \\
\text { Inches }\end{array}$ & 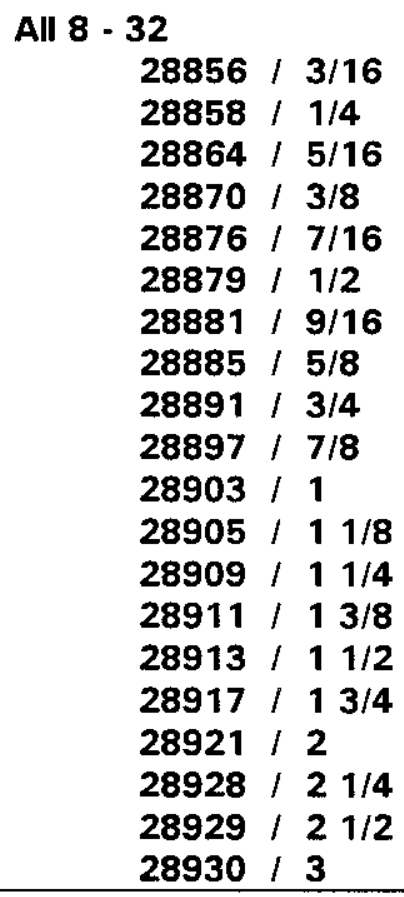 & 1, IN & $x$ \\
\hline $\begin{array}{l}\text { Pan Head Phillips Drive } \\
\text { Machine Screws - Size } \\
\text { - Thread, Part Number } \\
\text { / Nominal Length, } \\
\text { Inches }\end{array}$ & 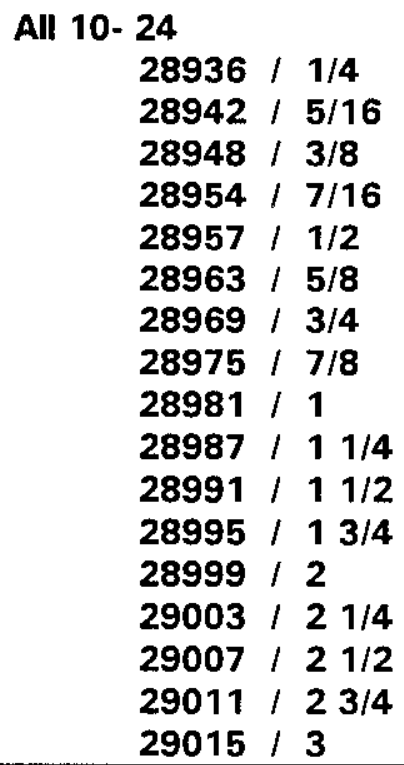 & 1, IN & $x$ \\
\hline $\begin{array}{l}\text { Pan Head Phillips Drive } \\
\text { Machine Screws - Size } \\
\text { - Thread, Part Number } \\
\text { / Nominal Length, } \\
\text { Inches }\end{array}$ & $\begin{aligned} & \text { All } 10- 32 \\
& 29055 / 5 / 8 \\
& 29073 / 1\end{aligned}$ & 1, IN & $x$ \\
\hline $\begin{array}{l}\text { Manufacturer, All Nylon Insert } \\
\text { Lock Nuts, Page } 104\end{array}$ & Fastenal & 1, IN & $x$ \\
\hline
\end{tabular}




\begin{tabular}{|l|l|}
\hline Commercial Grade Item Upgrade Dedication Form & SNF-5102 Rev. 1 \\
\hline $\begin{array}{l}\text { ECN No. NA CGI No. CGI-SNF-D-93-2-C1-056 } \\
\text { Title: CONDUIT FASTENERS }\end{array}$ & Page 8 of 33 \\
\hline
\end{tabular}

\begin{tabular}{|c|c|c|c|}
\hline $\begin{array}{l}\text { Part Number - Size - } \\
\text { Thread }\end{array}$ & $\begin{array}{cc}37010 & 6-32 \\
37012 & 8-32 \\
37014 & 10-24 \\
\end{array}$ & 1, IN & $\mathbf{x}$ \\
\hline $\begin{array}{l}\text { Manufacturer, All Size Hex Full } \\
\text { Nuts, Page } 101\end{array}$ & Fastenal & 1, IN & $\mathbf{x}$ \\
\hline $\begin{array}{l}\text { Part Number - Size - } \\
\text { Thread }\end{array}$ & $\begin{array}{l}36020 \\
36024 \\
36028 \\
\end{array}$ & 1, IN & $\mathbf{x}$ \\
\hline $\begin{array}{l}\text { Manufacturer, All Size SAE Flat } \\
\text { Washers, Page } 115\end{array}$ & Fastenal & 1, IN & $\mathbf{x}$ \\
\hline $\begin{array}{l}\text { Part Number - Size - } \\
\text { Nominal OD/ID, Inches }\end{array}$ & $\begin{array}{l}33070-6-3 / 8 / 5 / 32 \\
33072-8-7 / 16 / 3 / 16 \\
33074-10-1 / 2 / 7 / 32 \\
\end{array}$ & 1, IN & $\mathbf{x}$ \\
\hline $\begin{array}{l}\text { Manufacturer, All Size Medium } \\
\text { Split Lock Washers, Page } 116\end{array}$ & Fastenal & 1, IN & $\mathbf{x}$ \\
\hline Part Number - Size & $\begin{array}{rr}33610 & -6 \\
33612 & -8 \\
33614 & -10 \\
\end{array}$ & 1, IN & $\mathbf{x}$ \\
\hline \multicolumn{4}{|c|}{ 2. Physical Critical Characteristics (for reasonable assurance that the ltem delivered is the Item specified) } \\
\hline $\begin{array}{l}\text { Fender, Flat, and Lock Washers, } \\
\text { Material }\end{array}$ & Zinc Coated Steel (Note 3) & $\begin{array}{l}1, \mathrm{IN} \\
1, \mathrm{~T}\end{array}$ & $\mathbf{x}$ \\
\hline Hex Nuts, Material & Zinc Coated Steel (Note 3) & $\begin{array}{l}1, \text { IN } \\
1, T\end{array}$ & $\mathbf{x}$ \\
\hline Hex Head Bolts, Material & Zinc Coated Steel (Note 3) & $\begin{array}{l}1, \text { IN } \\
1, T\end{array}$ & $\mathbf{x}$ \\
\hline $\begin{array}{l}\text { Fasteners, } \\
\text { PS } 146 \text { Continuous } \\
\text { Threaded Rod, Material } \\
\text { PS RS Clamping Nut with } \\
\text { Regular Spring, Material } \\
\end{array}$ & $\begin{array}{l}\text { Steel (Note 3) } \\
\text { Steel (Note 3) }\end{array}$ & $\begin{array}{l}1, \mathrm{IN} \\
1, \mathrm{~T}\end{array}$ & $\mathbf{x}$ \\
\hline $\begin{array}{l}\text { All Size Pan Head Phillips Drive } \\
\text { Machine Screws, Material }\end{array}$ & Zinc Plated Steel (Note 3) & $\begin{array}{l}1, \text { IN } \\
1, T\end{array}$ & $\mathbf{x}$ \\
\hline $\begin{array}{l}\text { All Size Nylon Insert Lock Nuts, } \\
\text { Material }\end{array}$ & Zinc Plated Steel (Note 3) & $\begin{array}{l}1, \mathrm{IN} \\
1, \mathrm{~T}\end{array}$ & $x$ \\
\hline All Size Hex Full Nuts, Material & Zinc Plated Steel (Note 3) & $\begin{array}{l}1, \text { IN } \\
1, T\end{array}$ & $\mathbf{x}$ \\
\hline $\begin{array}{l}\text { All Size SAE Flat Washers, } \\
\text { Material }\end{array}$ & Zinc Plated Steel (Note 3) & $\begin{array}{l}1, \text { IN } \\
1, T\end{array}$ & $x$ \\
\hline
\end{tabular}




\begin{tabular}{|l|l|}
\hline Commercial Grade Item Upgrade Dedication Form & SNF-5102 Rev. 1 \\
\hline $\begin{array}{l}\text { ECN No. NA } \\
\text { Title: CONDUIT FASTENERS }\end{array}$ & Page 9 of 33 \\
\hline
\end{tabular}

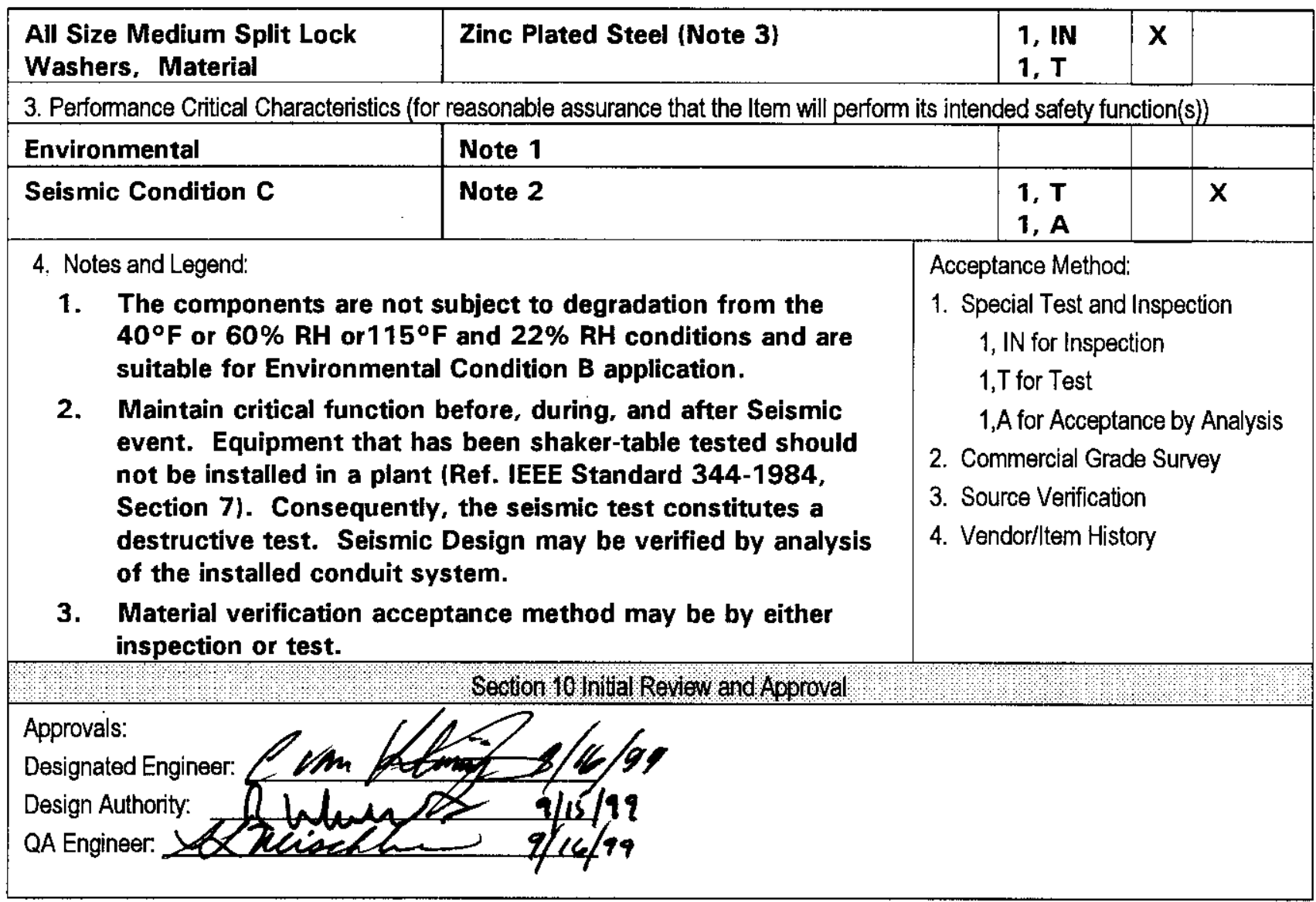




\begin{tabular}{|l|l|}
\hline Commercial Grade Item Upgrade Dedication Form & SNF-5102 Rev. 1 \\
\hline $\begin{array}{l}\text { ECN No. NA CGI No. CGI-SNF-D-93-2-C1-056 } \\
\text { Title: CONDUIT FASTENERS }\end{array}$ & Page 10 of 33 \\
\hline
\end{tabular}

\begin{tabular}{|c|c|c|}
\hline \multicolumn{3}{|c|}{$\begin{array}{c}\text { WORKSHEET } 1 \\
\text { DETERMINATION OF FALUURE MECHANISMS/MODES }\end{array}$} \\
\hline \multicolumn{3}{|c|}{ Soction / } \\
\hline $\begin{array}{l}\text { Typical Failure } \\
\text { Mechanisms }\end{array}$ & Definition & $\begin{array}{l}\text { Applicable to Component } \\
\text { under Evaluation }\end{array}$ \\
\hline Fracture & $\begin{array}{l}\text { Separation of a solid accompanied by little or no } \\
\text { macroscopic plastic deformation. }\end{array}$ & $\begin{array}{l}\text { Yes [X] No [ ]; If Yes, indicate failure } \\
\text { Mode: Fracture or material fatigue-loss of } \\
\text { structural protection to wiring }\end{array}$ \\
\hline Corrosion & $\begin{array}{l}\text { The gradual deterioration of a material due to } \\
\text { chemical or electrochemical reactions, such as } \\
\text { oxidation, between the material and its environment. }\end{array}$ & $\begin{array}{l}\text { Yes [ ] No [X]; If Yes, indicate failure } \\
\text { Mode }\end{array}$ \\
\hline Erosion & $\begin{array}{l}\text { Destruction of materials by the abrasive action of } \\
\text { moving fluids, usually accelerated by the presence } \\
\text { of solid particles carried with the fluid. }\end{array}$ & $\begin{array}{l}\text { Yes [ ] No [X]; If Yes, indicate failure } \\
\text { Mode }\end{array}$ \\
\hline Open Circuit & $\begin{array}{l}\text { An electrical circuit that is unintentionally broken so } \\
\text { that there is no complete path for current flow. }\end{array}$ & $\begin{array}{l}\text { Yes [ ] No [ X]; If Yes, indicate failure } \\
\text { Mode }\end{array}$ \\
\hline Short Circuit & $\begin{array}{l}\text { An abnormal connection by which an electrical } \\
\text { current is connected to ground, or to some } \\
\text { conducting body, resulting in excessive current flow. }\end{array}$ & $\begin{array}{l}\text { Yes [ ] No [ } \mathbf{X} \text { ]; If Yes, indicate failure } \\
\text { Mode }\end{array}$ \\
\hline Blockage & $\begin{array}{l}\text { Clogging of a filtering medium resulting in the } \\
\text { inability to perform its purification function or } \\
\text { blockage of flow. }\end{array}$ & $\begin{array}{l}\text { Yes [ ] No [X]; If Yes, indicate failure } \\
\text { Mode }\end{array}$ \\
\hline Seizure & $\begin{array}{l}\text { Binding of a normally moving item through excessive } \\
\text { pressure, temperature, friction, jamming. }\end{array}$ & $\begin{array}{l}\text { Yes [ ] No [X]; If Yes, indicate failure } \\
\text { Mode }\end{array}$ \\
\hline $\begin{array}{l}\text { Unacceptable } \\
\text { Vibration }\end{array}$ & $\begin{array}{l}\text { Mechanical oscillations produced are beyond the } \\
\text { defined permissible limits due to unbalancing, poor } \\
\text { support, or rotation at critical speeds. }\end{array}$ & $\begin{array}{l}\text { Yes [ ] No [X]; If Yes, indicate failure } \\
\text { Mode }\end{array}$ \\
\hline Loss of Properties & $\begin{array}{l}\text { A loss of mechanical and physical properties of a } \\
\text { material due to exposure to high temperatures, } \\
\text { radiation exposure. }\end{array}$ & $\begin{array}{l}\text { Yes [ ] No [X]; If Yes, indicate failure } \\
\text { Mode }\end{array}$ \\
\hline Excess Strain & $\begin{array}{l}\text { Under the action of excessive extemal forces the } \\
\text { material of the part has been deformed or distorted. }\end{array}$ & $\begin{array}{l}\text { Yes [X] No [ ]; If Yes, indicate failure } \\
\text { Mode; Material fatigue leads to loss of } \\
\text { structural support and enclosure of wiring }\end{array}$ \\
\hline Mechanical Cröp & $\begin{array}{l}\text { From prolonged exposure to high temperature and } \\
\text { stress, the object will show a slow change in its } \\
\text { physical (shape and dimension) and mechanical } \\
\text { characteristics. }\end{array}$ & $\begin{array}{l}\text { Yes [ ] No [X]; If Yes, indicate failure } \\
\text { Mode }\end{array}$ \\
\hline Ductile Fracture & $\begin{array}{l}\text { Fracture characterized by tearing of metal } \\
\text { accompanied by appreciable gross plastic } \\
\text { deformation. }\end{array}$ & $\begin{array}{l}\text { Yes [X] No [ ]; If Yes, indicate failure } \\
\text { Mode: Fracture or material fatigue - loss of } \\
\text { structural support and protection of wiring }\end{array}$ \\
\hline \multirow{2}{*}{\multicolumn{3}{|c|}{ Section 2 Additional Fallure Modes Applicabie to the Gomponent Under Evaluation }} \\
\hline & & \\
\hline 2. & & \\
\hline
\end{tabular}




\begin{tabular}{|l|l|}
\hline Commercial Grade Item Upgrade Dedication Form & SNF-5102 Rev. 1 \\
\hline $\begin{array}{l}\text { ECN No. NA CGI No. CGI-SNF-D-93-2-C1-056 } \\
\text { Title: CONDUIT FASTENERS }\end{array}$ & Page 11 of 33 \\
\hline
\end{tabular}

\section{CHECKLIST 1}

\section{ACCEPTANCE METHOD 1}

SPECIAL TEST/INSPECTION VERIFICATION

\begin{tabular}{|c|c|c|c|c|}
\hline & & & & SEGTION 1 \\
\hline $\begin{array}{l}\text { Item D } \\
\text { Wiring } \\
\text { System }\end{array}$ & $\begin{array}{l}\text { Condu } \\
\text { Co3-2 }\end{array}$ & $\begin{array}{l}\text { Fast } \\
\text { it. }\end{array}$ & ners for Safety Class & $\begin{array}{l}\text { Equip \#: NA } \\
\text { Procurement and/or Model \#: See Catalog Numbers in } \\
\text { Section } 8 \text { and Characteristics in Section } 9 .\end{array}$ \\
\hline $\begin{array}{l}\text { Variou } \\
\text { P.O. \# }\end{array}$ & $\begin{array}{l}\text { Mant } \\
\text { - See }\end{array}$ & $\begin{array}{l}\text { facture } \\
\text { Sectio }\end{array}$ & $\begin{array}{l}\text { (Address/Phone): } \\
\text { s } 8 \text { and } 9 .\end{array}$ & Supplier (Address/Phone): \\
\hline & & & CTION2 CRIICAL CHAR & ERSSTICS TO BE VERFIEO BY MGTHOO / \\
\hline Insp & Test & $\begin{array}{l}\text { Past- } \\
\text { Test }\end{array}$ & & \\
\hline$[\mathbf{X}]$ & [ ] & l l & 1. Fender, Flat, and & k Washers \\
\hline$[\mathbf{X}]$ & {$[1$} & 11 & 1. Hex Nuts & \\
\hline$[\mathbf{X}]$ & l 1 & 11 & 2. Hex Head Bolts & \\
\hline$[\mathbf{X}]$ & [ ] & [ ] & 3. Fasteners & \\
\hline$[\mathbf{X}]$ & [ 1 & I l & 4. Pan Head Phillips & ve Machine Screws \\
\hline$[\mathbf{X}]$ & [ ] & [ ] & 5. Nylon Insert Lock & \\
\hline$[\mathbf{X}]$ & {$[$ ] } & 11 & 6. Hex Full Nuts & \\
\hline$[\mathbf{X}]$ & [ ] & {$[1]$} & 7. SAE Flat Washer: & \\
\hline$[\mathbf{X}]$ & [ ] & [ ] & 8. Medium Split Loc & lashers \\
\hline$[\mathbf{X}]$ & {$[\mathbf{X}]$} & [ ] & $\begin{array}{l}\text { 9. Material, Fender, } \\
\text { inspection or by }\end{array}$ & , and Lock Washers (Verification may be by either \\
\hline$[\mathbf{X}]$ & {$[\mathbf{X}]$} & [ ] & 10. Material, Hex Nu & /erification may be by either inspection or by test) \\
\hline$[\mathbf{X}]$ & {$[\mathbf{X}]$} & {$[$ [ ] } & $\begin{array}{l}\text { 11. Material, Hex He } \\
\text { test) }\end{array}$ & Its (Verification may be by either inspection or by \\
\hline$[\mathbf{X}]$ & {$[\mathbf{X}]$} & {$[1]$} & 12. Material, Fastene & Verification may be by either inspection or by test) \\
\hline$[\mathbf{X}]$ & {$[\mathbf{X}]$} & {$[$ ] } & $\begin{array}{l}\text { 13. Material, Pan He: } \\
\text { either inspection }\end{array}$ & $\begin{array}{l}\text { hillips Drive Machine Screws (Verification may be by } \\
\text { y test) }\end{array}$ \\
\hline$[\mathbf{X}]$ & {$[\mathbf{X}]$} & [ l $]$ & $\begin{array}{l}\text { 14. Material, Nylon Ir } \\
\text { by test) }\end{array}$ & Lock Nuts (Verification may be by either inspection or \\
\hline$[\mathbf{X}\}$ & {$[\mathbf{X}]$} & {$[1$} & 15. Material, Hex Ful & ts (Verification may be by elther inspection or by test) \\
\hline$[\mathbf{X}]$ & {$[\mathbf{X}]$} & [ ] & $\begin{array}{l}\text { 16. Material, SAE Fla } \\
\text { test) }\end{array}$ & shers (Verification may be by either inspection or by \\
\hline
\end{tabular}




\begin{tabular}{|l|l|}
\hline Commercial Grade Item Upgrade Dedication Form & SNF-5102 Rev. 1 \\
\hline $\begin{array}{l}\text { ECN No. } \text { NA CGI No. CGI-SNF-D-93-2-C1-056 } \\
\text { Title: CONDUIT FASTENERS }\end{array}$ & Page 12 of 33 \\
\hline
\end{tabular}

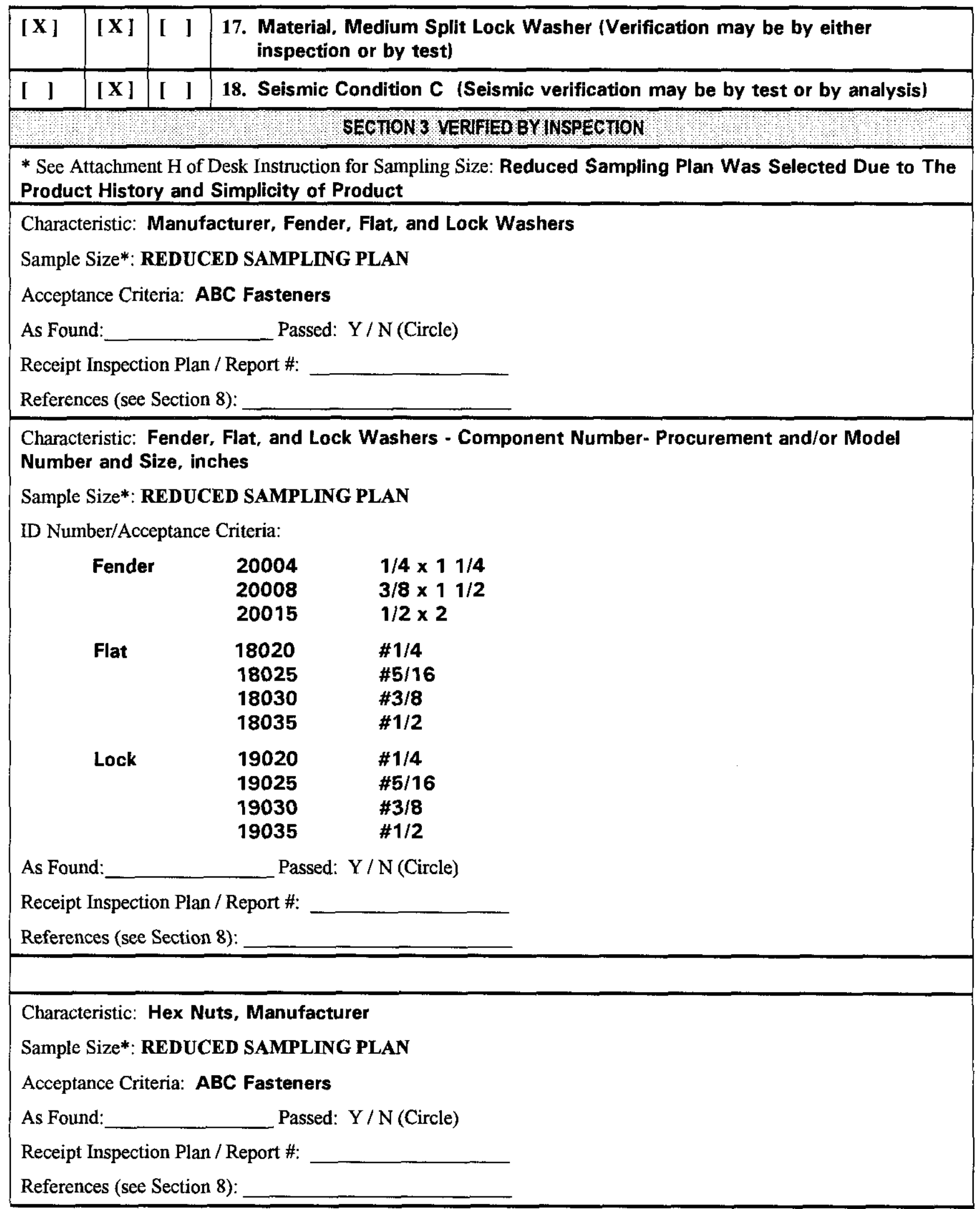




\begin{tabular}{|l|l|}
\hline Commercial Grade Item Upgrade Dedication Form & SNF-5102 Rev. 1 \\
\hline $\begin{array}{l}\text { ECN No. NA CGI No. CGI-SNF-D-93-2-C1-056 } \\
\text { Title: CONDUIT FASTENERS }\end{array}$ & Page 13 of 33 \\
\hline
\end{tabular}

Characteristic: Hex Nuts - Component Number- Procurement and/or Model Number and Size/Thread, inches

\section{Sample Size*: REDUCED SAMPLING PLAN}

ID Number - Size / Acceptance Criteria:

$\begin{array}{ll}17025 & 1 / 4-20 \\ 17030 & 5 / 16-18 \\ 17035 & 3 / 8-16 \\ 17040 & 1 / 2-13\end{array}$

As Found: Passed: Y/N (Circle)

Receipt Inspection Plan / Report \#:

References (see Section 8):

Characteristic: Hex Head Bolts - Manufacturer

Sample Size*: REDUCED SAMPLING PLAN

Acceptance Criteria: ABC Fasteners

As Found: Passed: Y/N (Circle)

Receipt Inspection Plan / Report \#:

References (see Section 8):

Characteristic: Hex Head Bolts - Component Number- Procurement and/or Model Number - Size/Thread and Nominal Length, inches

Sample Size*: REDUCED SAMPLING PLAN

ID Number -Size - Thread -Nominal Length / Acceptance Criteria:

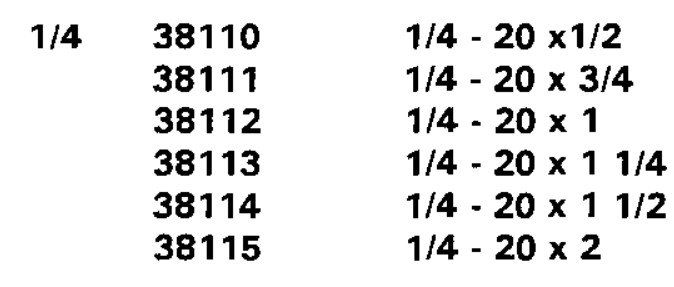

$\begin{array}{lll}5 / 16 & 38126 & 5 / 16-18 \times 3 / 4 \\ 38127 & 5 / 16-18 \times 1 \\ 38128 & 5 / 16-18 \times 11 / 4\end{array}$

$3 / 8 \quad 38141 \quad 3 / 8-16 \times 3 / 4$

$38142 \quad 3 / 8-16 \times 1$

$38143 \quad 3 / 8-16 \times 11 / 4$

$38144 \quad 3 / 8-16 \times 11 / 2$

As Found: Passed: Y/N (Circle)

Receipt Inspection Plan / Report \#:

References (see Section 8): 


\begin{tabular}{|l|l|}
\hline Commercial Grade Item Upgrade Dedication Form & SNF-5102 Rev. 1 \\
\hline $\begin{array}{l}\text { ECN No. NA CGI No. CGI-SNF-D-93-2-C1-056 } \\
\text { Title: CONDUIT FASTENERS }\end{array}$ & Page 14 of 33 \\
\hline
\end{tabular}

Characteristic: Manufacturer, Fasteners

Sample Size*: REDUCED SAMPLING PLAN

Acceptance Criteria: Powerstrut

As Found: Passed: Y / N (Circle)

Receipt Inspection Plan / Report \#:

References (see Section 8):

Characteristic: Fasteners - Component Number-Procurement and/or Model Number and Size, inches Sample Size*: REDUCED SAMPLING PLAN

ID Number - Size/Acceptance Criteria: PS 146 Continuous Threaded Rod / Nominal 3/8"

Size

/ Nominal 1/2"

/ Nominal $\mathbf{5} / \mathbf{8}^{\mathrm{n}}$

/ Nominal 3/4"

PS RS Clamping Nut with Regular Spring / 1/4" x 20

Size $\times$ Thread $\quad / 3 / 8^{\prime \prime} \times 16$

$/ 1 / 2 " \times 13$

$/ 5 / 8^{\prime \prime} \times 11$

$13 / 4^{n} \times 10$

$17 / 8 " \times 9$

As Found: Passed: Y / N (Circle)

Receipt Inspection Plan / Report \#:

References (see Section 8):

Characteristic: Manufacturer, All Pan Head Phillips Drive Machine Screws

Sample Size*: REDUCED SAMPLING PLAN

Acceptance Criteria: Fastenal

As Found: Passed: Y / N (Circle)

Receipt Inspection Plan / Report \#:

References (see Section 8): 


\begin{tabular}{|l|l|}
\hline Commercial Grade Item Upgrade Dedication Form & SNF-5102 Rev. 1 \\
\hline $\begin{array}{l}\text { ECN No. NA } \\
\text { Title: } \text { CONDUIT FASTENERS }\end{array}$ & Page 15 of 33 \\
\hline
\end{tabular}

Characteristic: Pan Head Phillips Drive Machine Screws

Sample Size*: REDUCED SAMPLING PLAN

ID All Size - Thread Part Number / Nominal Length, Inches / Acceptance Criteria:

\begin{tabular}{|c|c|c|}
\hline All 6 - 32 & $\begin{array}{l}28780 \\
28781 \\
28783 \\
28789 \\
28795 \\
28801 \\
28804 \\
28806 \\
28810 \\
28816 \\
28822 \\
28828 \\
28830 \\
28834 \\
28836 \\
28838 \\
28840 \\
28842 \\
28846 \\
28852 \\
28853 \\
28854\end{array}$ & 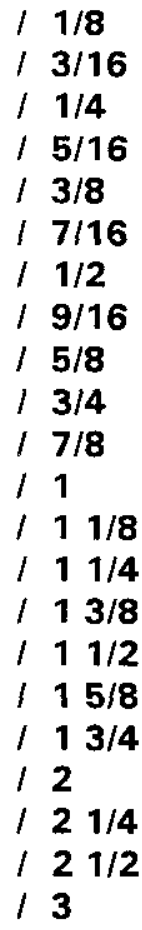 \\
\hline
\end{tabular}

As Found: Passed: Y / N (Circle)

Receipt Inspection Plan / Report \#:

References (see Section 8): Machine Screws - Fastenal Catalog, Pages 76 and 77 


\begin{tabular}{|l|l|}
\hline Commercial Grade Item Upgrade Dedication Form & SNF-5102 Rev. 1 \\
\hline $\begin{array}{l}\text { ECN No. NA CGI No. CGI-SNF-D-93-2-C1-056 } \\
\text { Title: CONDUIT FASTENERS }\end{array}$ & Page 16 of 33 \\
\hline
\end{tabular}

Characteristic: Pan Head Phillips Drive Machine Screws

Sample Size*: REDUCED SAMPLING PLAN

ID All Size - Thread Part Number / Nominal Length, Inches / Acceptance Criteria:

$\begin{array}{cl:l}\text { All } 8 \text { - } 32 & 28856 & 3 / 16 \\ & 28858 & 1 / 4 \\ 28864 & 5 / 16 \\ 28870 & 3 / 8 \\ 28876 & 7 / 16 \\ 28879 & 1 / 2 \\ 28881 & 9 / 16 \\ 28885 & 5 / 8 \\ 28891 & 3 / 4 \\ 28897 & 7 / 8 \\ 28903 & 1 \\ 28905 & 11 / 8 \\ 28909 & 1 / 4 \\ 28911 & 13 / 8 \\ 28913 & 1 / 2 \\ 28917 & 13 / 4 \\ 28921 & 2 \\ 28928 & 21 / 4 \\ 28929 & 21 / 2 \\ 28930 & 3\end{array}$

As Found: Passed: $\mathrm{Y} / \mathrm{N}$ (Circle)

Receipt Inspection Plan / Report \#:

References (see Section 8): Machine Screws - Fastenal Catalog, Pages 76 and 77 


\begin{tabular}{|l|l|}
\hline Commercial Grade Item Upgrade Dedication Form & SNF-5102 Rev. 1 \\
\hline $\begin{array}{l}\text { ECN No. } \text { NA CGI No. CGI-SNF-D-93-2-C1-056 } \\
\text { Title: CONDUIT FASTENERS }\end{array}$ & Page 17 of 33 \\
\hline
\end{tabular}

\section{Characteristic: Pan Head Phillips Drive Machine Screws \\ Sample Size*: REDUCED SAMPLING PLAN \\ ID All Size - Thread Part Number / Nominal Length, Inches / Acceptance Criteria:}

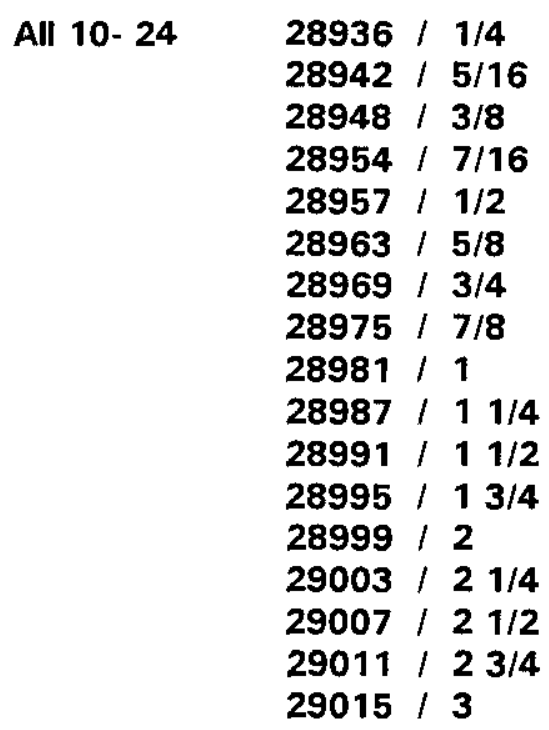

As Found: Passed: Y / N (Circle)

Receipt Inspection Plan / Report \#:

References (see Section 8): Machine Screws - Fastenal Catalog, Pages 76 and 77

Characteristic: Pan Head Phillips Drive Machine Screws

Sample Size*: REDUCED SAMPLING PLAN

ID All Size - Thread Part Number / Nominal Length, Inches / Acceptance Criteria:

All 10- $32 \quad 29055 / 5 / 8$

$29073 / 1$

As Found: Passed: Y / N (Circle)

Receipt Inspection Plan / Report \#:

References (see Section 8): Machine Screws - Fastenal Catalog, Pages 76 and 77

Characteristic: Manufacturer, All Nylon Insert Lock Nuts

Sample Size*: REDUCED SAMPLING PLAN

Acceptance Criteria: Fastenal

As Found: Passed: Y / N (Circle)

Receipt Inspection Plan / Report \#:

References (see Section 8): 


\begin{tabular}{|l|l|}
\hline Commercial Grade Item Upgrade Dedication Form & SNF-5102 Rev. 1 \\
\hline $\begin{array}{l}\text { ECN No. NA CGI No. CGI-SNF-D-93-2-C1-056 } \\
\text { Title: CONDUIT FASTENERS }\end{array}$ & Page 18 of 33 \\
\hline
\end{tabular}

\begin{tabular}{|c|c|}
\hline \\
\hline & \\
\hline \multicolumn{2}{|c|}{$\begin{array}{l}\text { Characteristic: Nylon Insert Lock } \\
\text { Sample Size*: REDUCED SAMP }\end{array}$} \\
\hline D Part Number & $\mathrm{ze}-\mathrm{Thr}$ \\
\hline $\begin{array}{l}3701 \\
3701\end{array}$ & \\
\hline $\begin{array}{l}37012 \\
37014\end{array}$ & \\
\hline
\end{tabular}

As Found: Passed: Y / N (Circle)

Receipt Inspection Plan / Report \#:

References (see Section 8): Nylon Insert Lock Nuts, Fastenal Catalog, Page 104

Characteristic: Manufacturer, All Size Hex Full Nuts

Sample Size*: REDUCED SAMPLING PLAN

Acceptance Criteria: Fastenal

As Found: Passed: Y / N (Circle)

Receipt Inspection Plan / Report \#:

References (see Section 8):

Characteristic: All Size .Hex Full Nuts

Sample Size*: REDUCED SAMPLING PLAN

ID - Part Number - Size-Thread / Acceptance Criteria:

$$
\begin{array}{cc}
36020 & -6-32 \\
36024 & -8-32 \\
36028 & -10-24
\end{array}
$$

As Found: Passed: $\mathrm{Y} / \mathrm{N}$ (Circle)

Receipt Inspection Plan / Report \#:

References (see Section 8): Hex Full Nuts, Fastenal Catalog, Page 101

Characteristic: Manufacturer, All Size SAE Flat Washers

Sample Size*: REDUCED SAMPLING PLAN

Acceptance Criteria: Fastenal

As Found: Passed: Y / N (Circle)

Receipt Inspection Plan / Report \#:

References (see Section 8): 


\begin{tabular}{|l|l|}
\hline Commercial Grade Item Upgrade Dedication Form & SNF-5102 Rev. 1 \\
\hline $\begin{array}{l}\text { ECN No. NA CGI No. CGI-SNF-D-93-2-C1-056 } \\
\text { Title: CONDUIT FASTENERS }\end{array}$ & Page 19 of 33 \\
\hline
\end{tabular}

Characteristic: SAE Flat Washers

Sample Size*: REDUCED SAMPLING PLAN

ID - Part Number - Size - Nominal OD/ID, Inches / Acceptance Criteria:

$33070-6-3 / 8 / 5 / 32$

$33072-8-7 / 16 / 3 / 16$

$33074 \cdot 10-1 / 2 / 7 / 32$

As Found: Passed: Y / N (Circle)

Receipt Inspection Plan / Report \#:

References (see Section 8): SAE Flat Washers, Fastenal Catalog, Page 115

Characteristic: Manufacturer, All Size Medium Split Lock Washers

Sample Size*: REDUCED SAMPLING PLAN

Acceptance Criteria: Fastenal

As Found: Passed: $\mathrm{Y} / \mathrm{N}$ (Circle)

Receipt Inspection Plan / Report \#:

References (see Section 8):

Characteristic: Medium Split Lock Washers

Sample Size*: REDUCED SAMPLING PLAN

ID - Part Number - Size / Acceptance Criteria:

$\begin{array}{rr}33610 & 6 \\ 33612 & 8 \\ 33614 & 10\end{array}$

As Found: Passed: Y / N (Circle)

Receipt Inspection Plan / Report \#:

References (see Section 8): Medium Split Lock Washers, Fastenal Catalog, Page 116

Characteristic: Material, Fender, Flat, and Lock Washers

Sample Size*: REDUCED SAMPLING PLAN

Acceptance Criteria: Zinc Coated Steel

As Found: Passed: Y / N (Circle)

Receipt Inspection Plan / Report \#:

References (see Section 8): 


\begin{tabular}{|l|l|}
\hline Commercial Grade Item Upgrade Dedication Form & SNF-5102 Rev. 1 \\
\hline $\begin{array}{l}\text { ECN No. NA CGI No. CGI-SNF-D-93-2-C1-056 } \\
\text { Title: CONDUIT FASTENERS }\end{array}$ & Page 20 of 33 \\
\hline
\end{tabular}

\section{Characteristic: Material, Hex Nuts \\ Sample Size*: REDUCED SAMPLING PLAN \\ Acceptance Criteria: Zinc Coated Steel \\ As Found: Passed: Y / N (Circle) \\ Receipt Inspection Plan / Report \#: \\ References (see Section 8): \\ Characteristic: Material, Hex Head Bolts \\ Sample Size*: REDUCED SAMPLING PLAN \\ Acceptance Criteria: Zinc Coated Steel \\ As Found: Passed: Y / N (Circle) \\ Receipt Inspection Plan / Report \#: \\ References (see Section 8):}

Characteristic: Material, Fasteners

Sample Size*: REDUCED SAMPLING PLAN

Acceptance Criteria:

PS 146 Continuous

Threaded Rod. Steel

PS RS Clamping Nut with

Regular Spring. Steel

As Found: Passed: Y / N (Circle)

Receipt Inspection Plan / Report \#:

References (see Section 8):

Characteristic: Material, All Pan Head Phillips Drive Machine Screws

Sample Size*: REDUCED SAMPLING PLAN

Acceptance Criteria: Zinc Plated Steel

As Found: Passed: Y / N (Circle)

Receipt Inspection Plan / Report \#:

References (see Section 8):

Characteristic: Material, All Nylon Insert Lock Nuts

Sample Size*: REDUCED SAMPLING PLAN

Acceptance Criteria: Zinc Plated Steel

As Found: Passed: Y / N (Circle)

Receipt Inspection Plan / Report \#:

References (see Section 8): 


\begin{tabular}{|l|l|}
\hline Commercial Grade Item Upgrade Dedication Form & SNF-5102 Rev. 1 \\
\hline $\begin{array}{l}\text { ECN No. NA CGI No. CGI-SNF-D-93-2-C1-056 } \\
\text { Title: CONDUIT FASTENERS }\end{array}$ & Page 21 of 33 \\
\hline
\end{tabular}

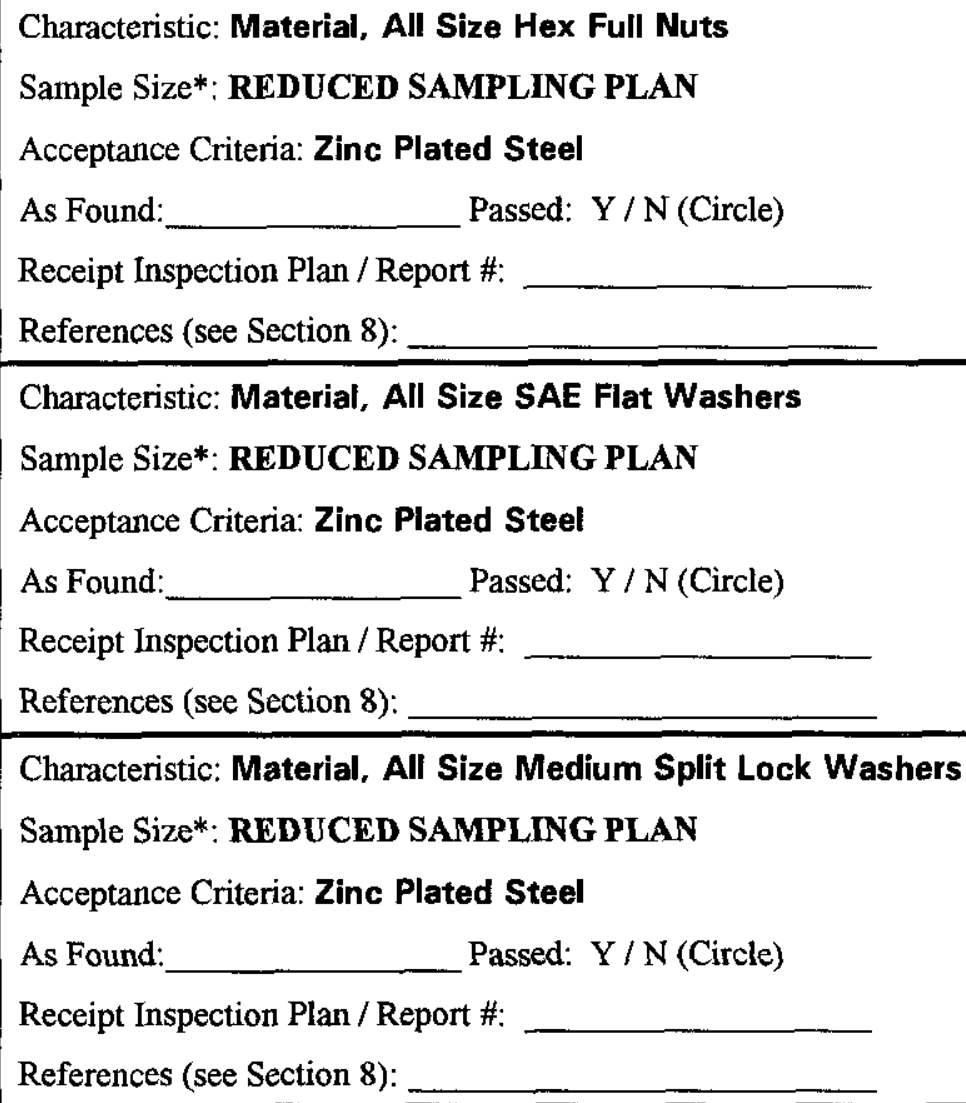

\section{SECTION 4 VERIFED BY SPECIAL TEST}

* See Attachment $\mathrm{H}$ of Desk Instruction for Sampling Size: NA

Characteristic for Test: Seismic Condition C - All Items

Acceptance Criteria: Maintain Critical Function Before, During, and After Seismic Event.

Sample Size*: Sample Size is NA because seismic verification of the installed conduit system with fittings, supports, etc, will be done by analysis. Physical testing is not anticipated. Actual Test Value:

Test Plan and Report \#: References (see Section 8): 


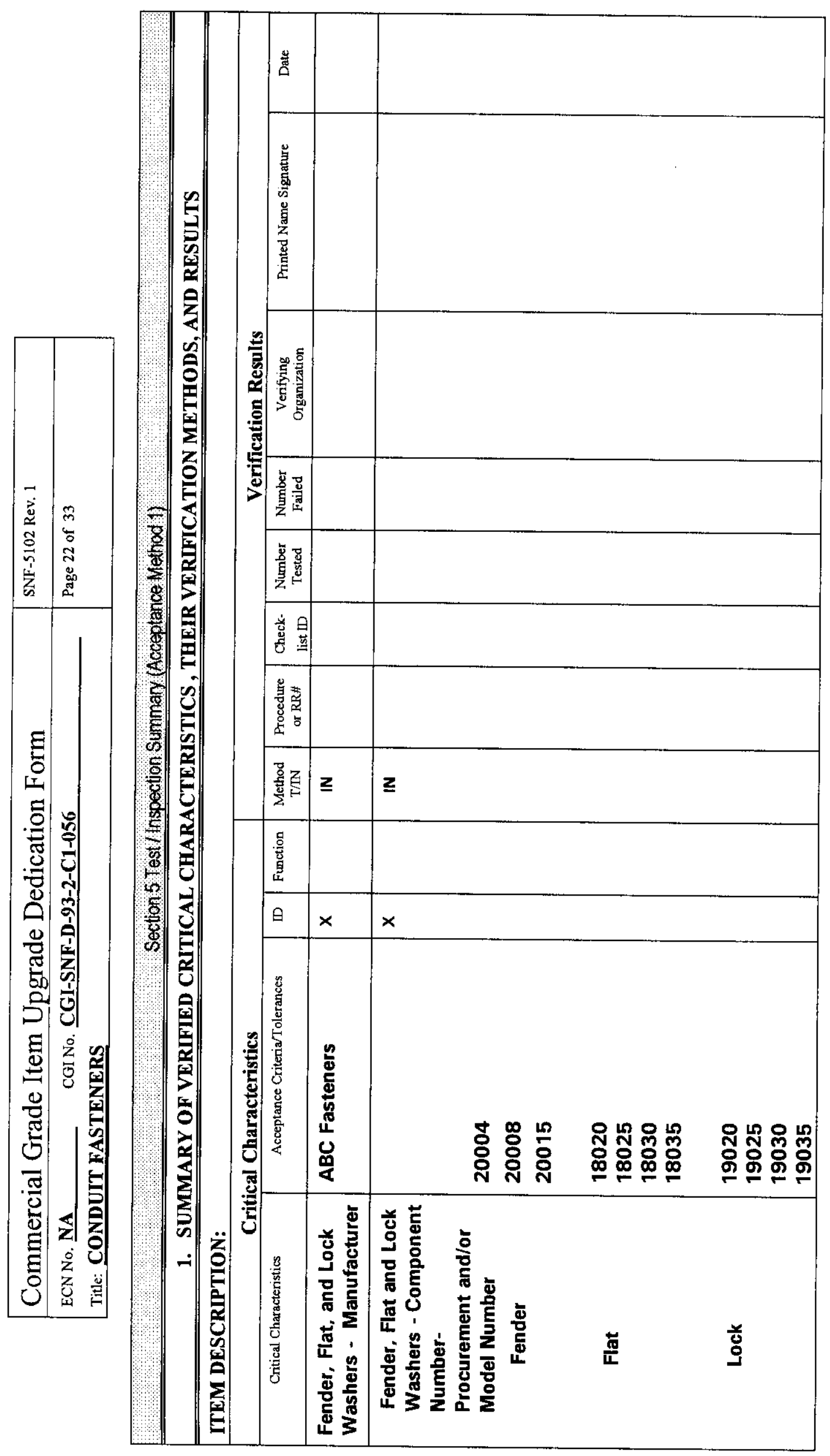

$\frac{\frac{9}{2}}{\frac{5}{2}}$ 


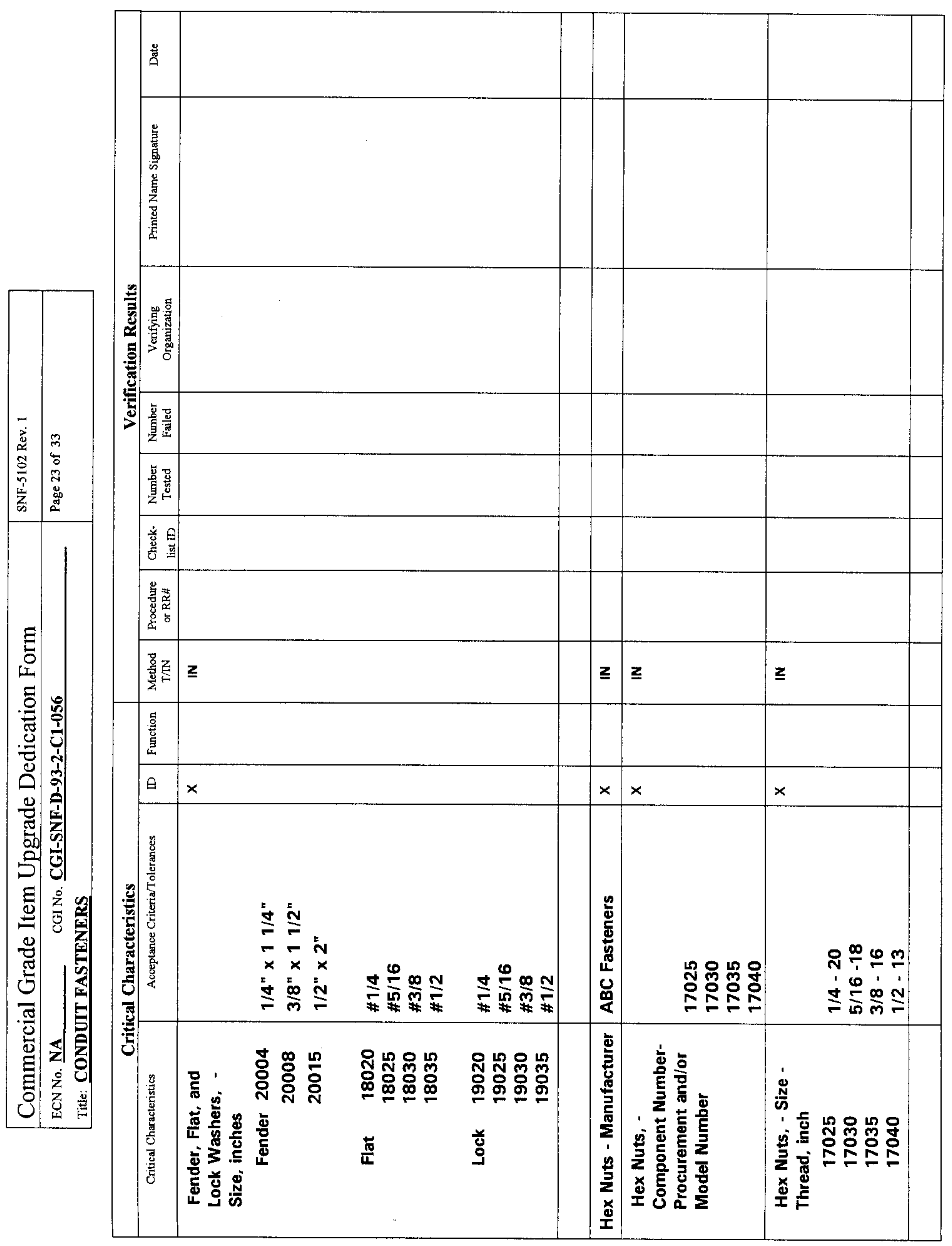




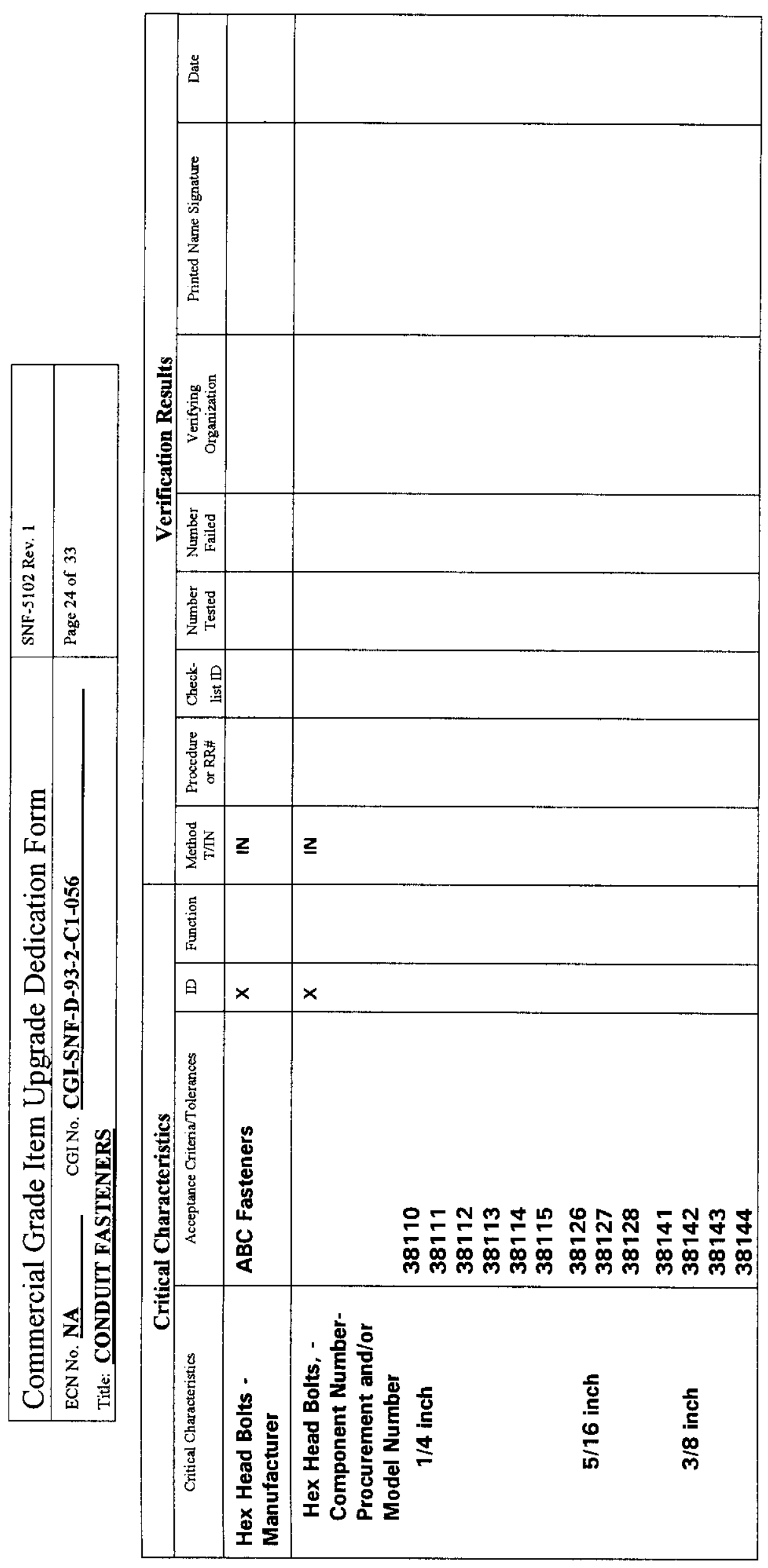




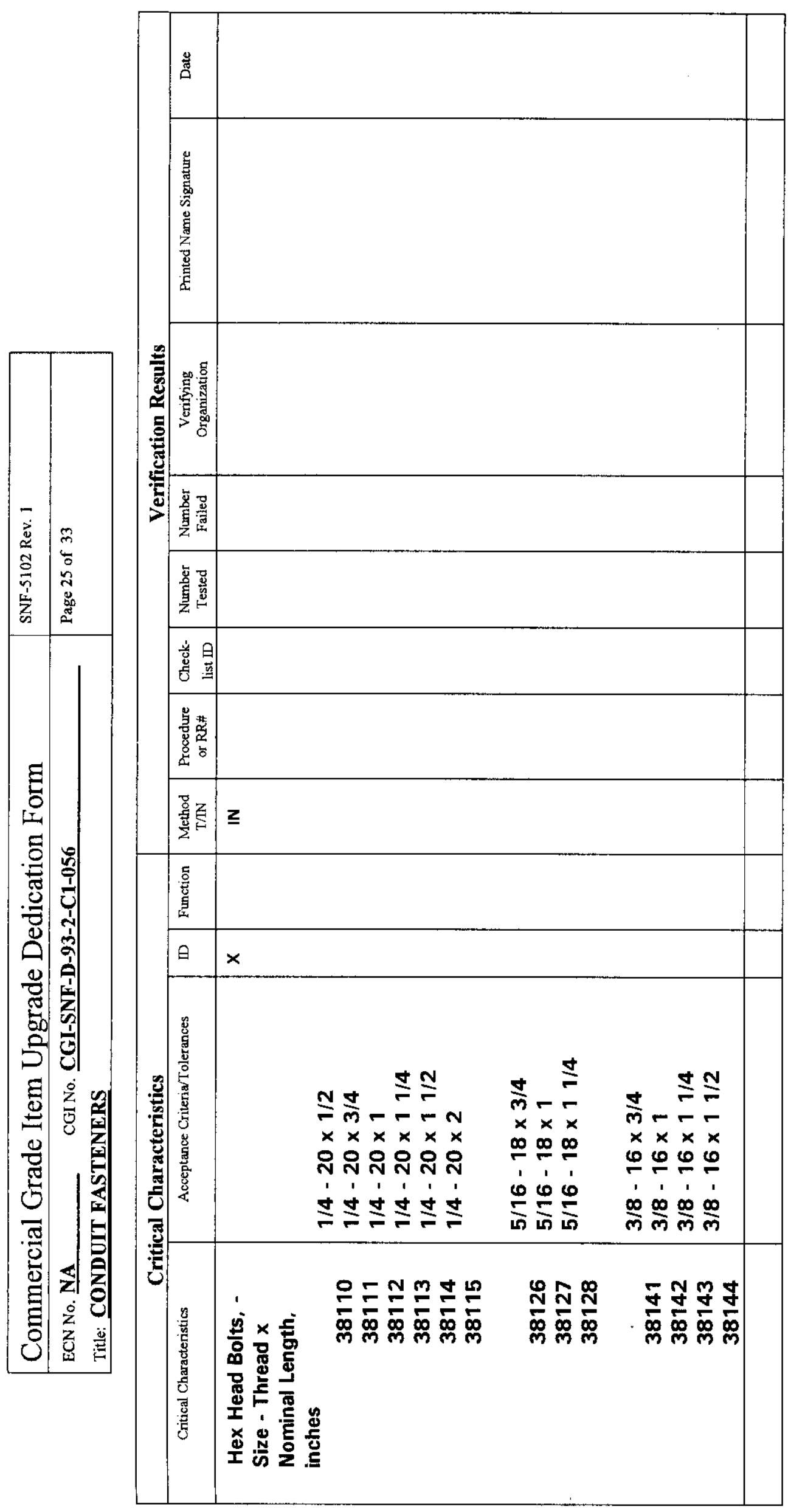




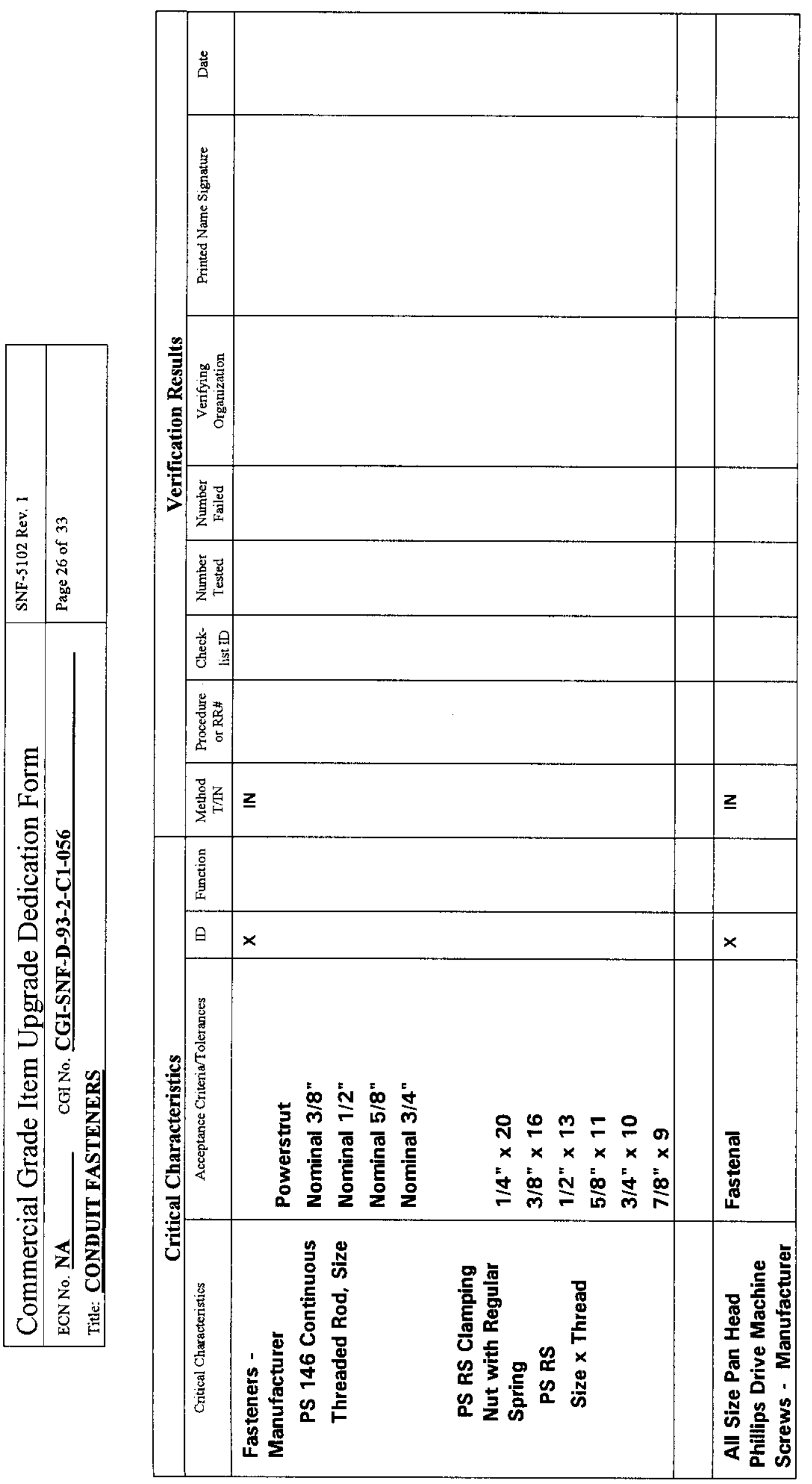




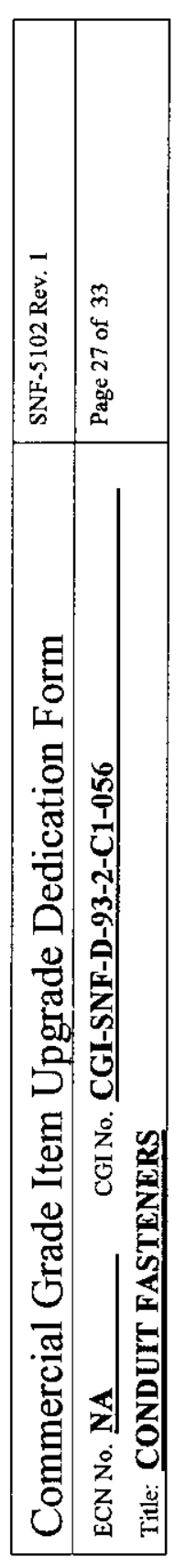

\begin{tabular}{|c|c|c|}
\hline$\frac{2}{2}$ & 善 & \\
\hline & 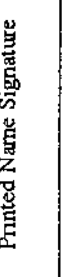 & \\
\hline 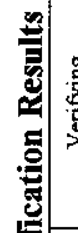 & & \\
\hline & $\frac{8}{2}$ & \\
\hline & 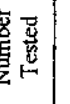 & \\
\hline & 递圆 & \\
\hline & & \\
\hline & 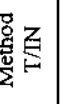 & $\underline{z}$ \\
\hline & & \\
\hline$E$ & & $x$ \\
\hline 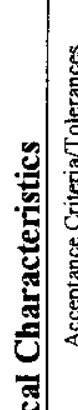 & & 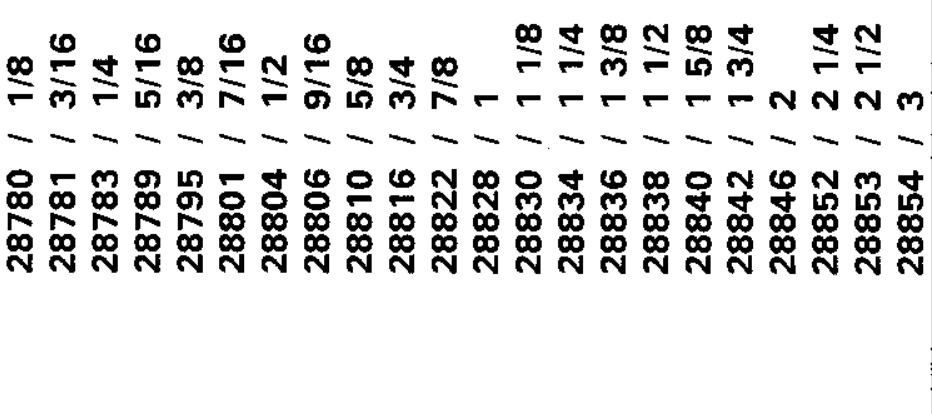 \\
\hline 它 & 造 & 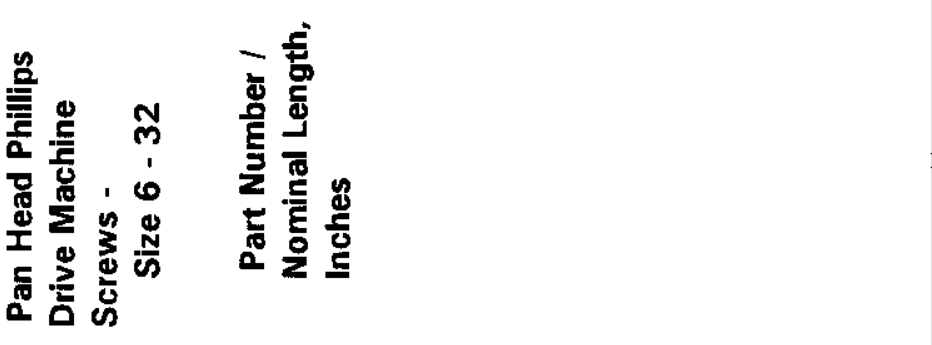 \\
\hline
\end{tabular}




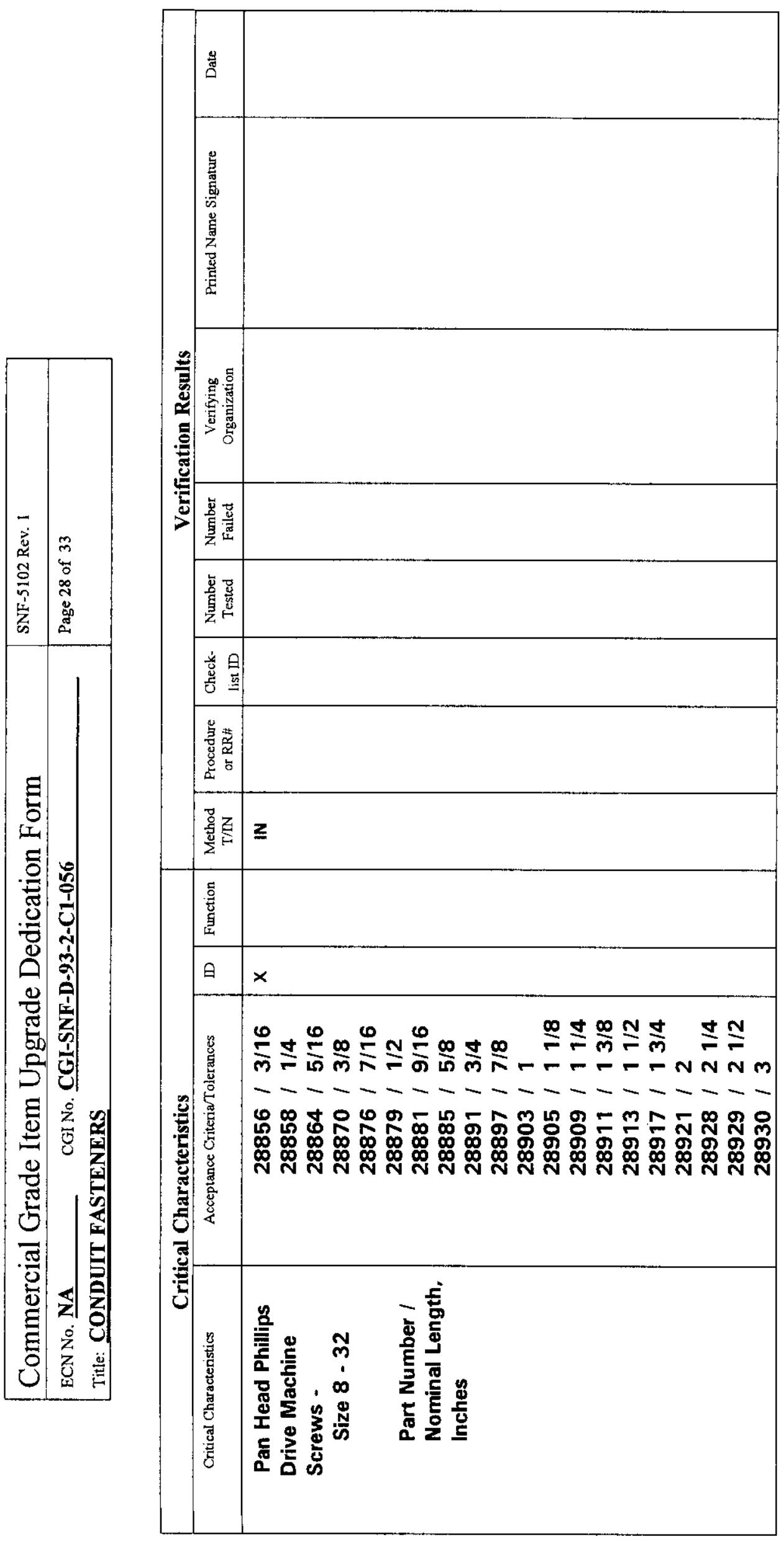




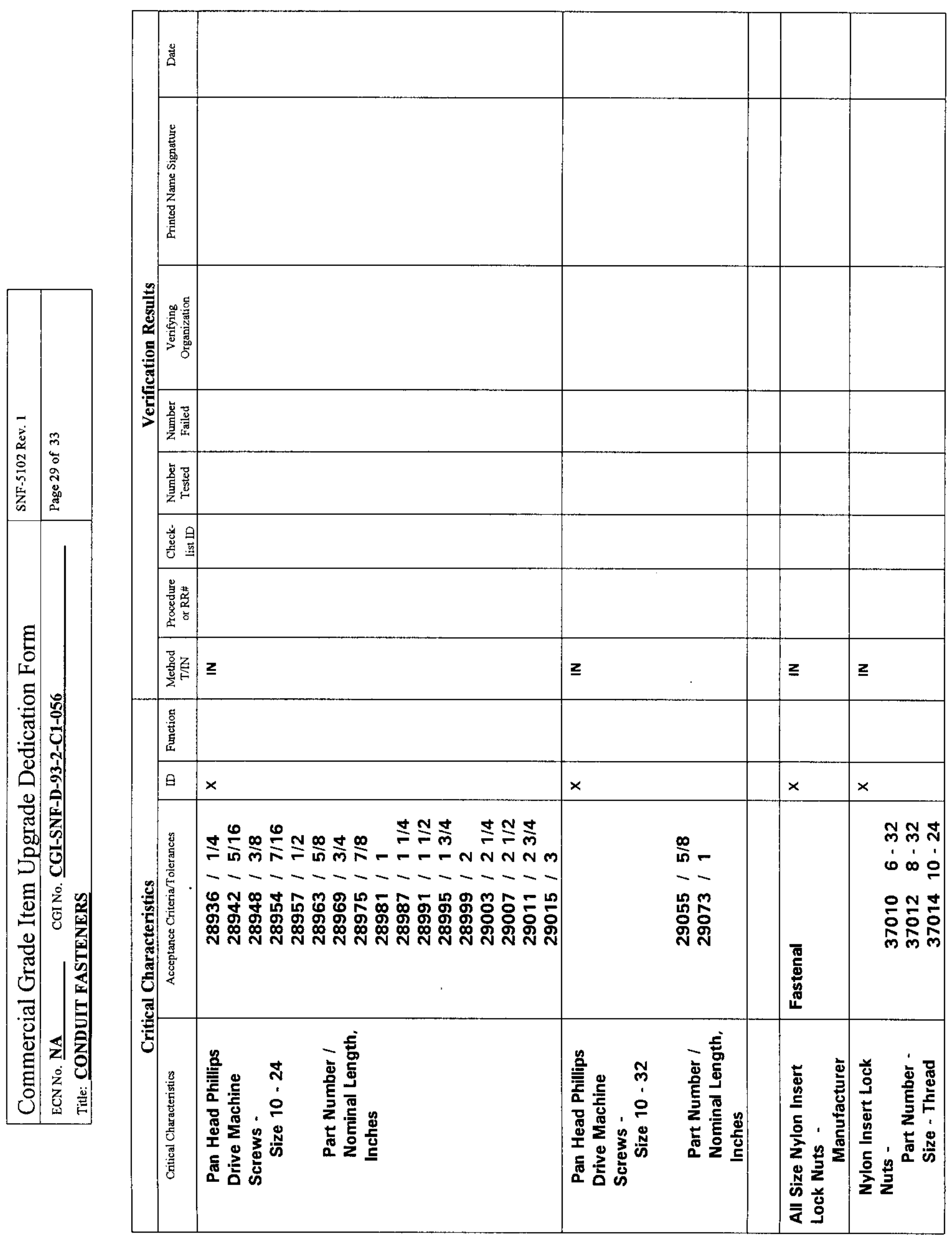




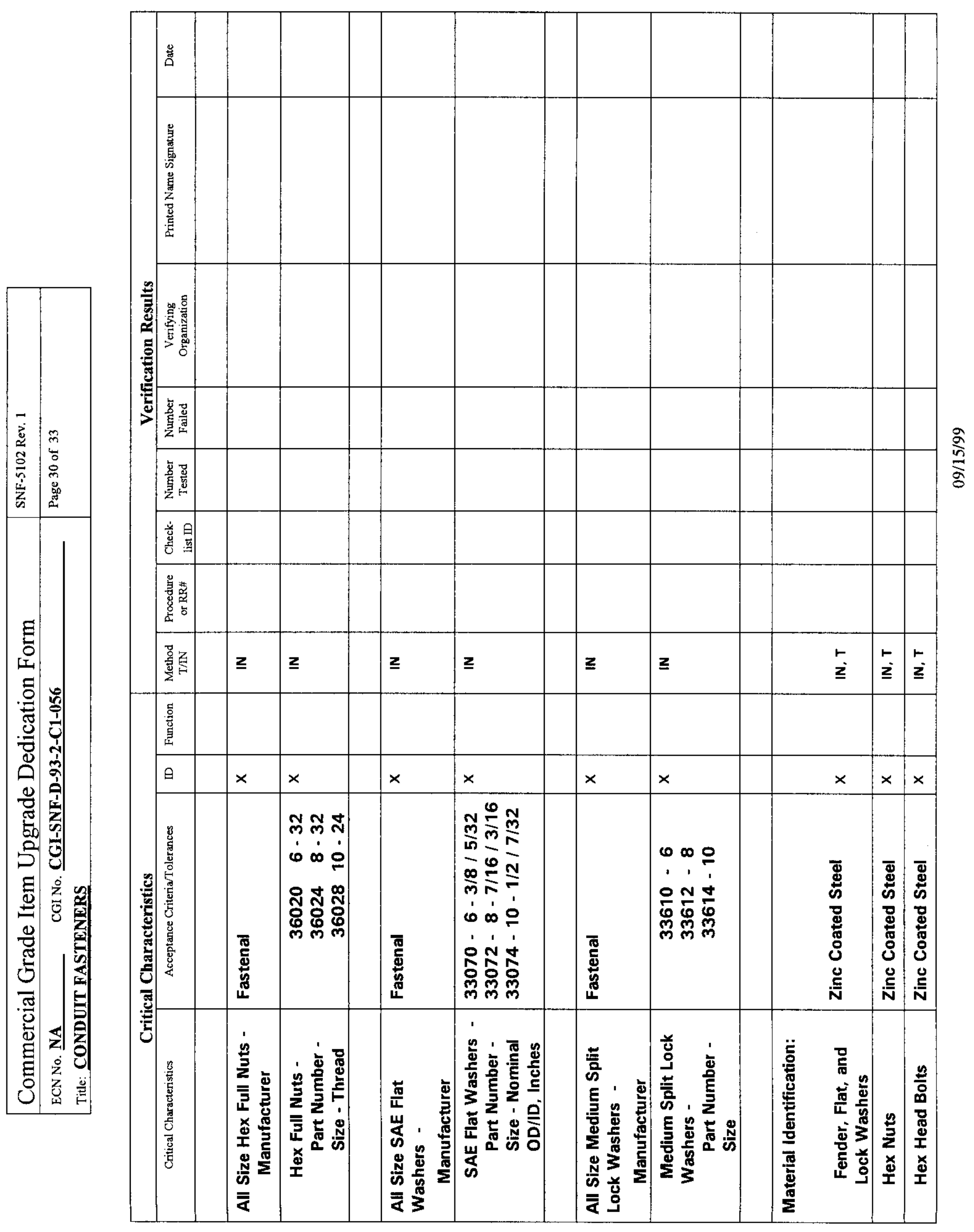




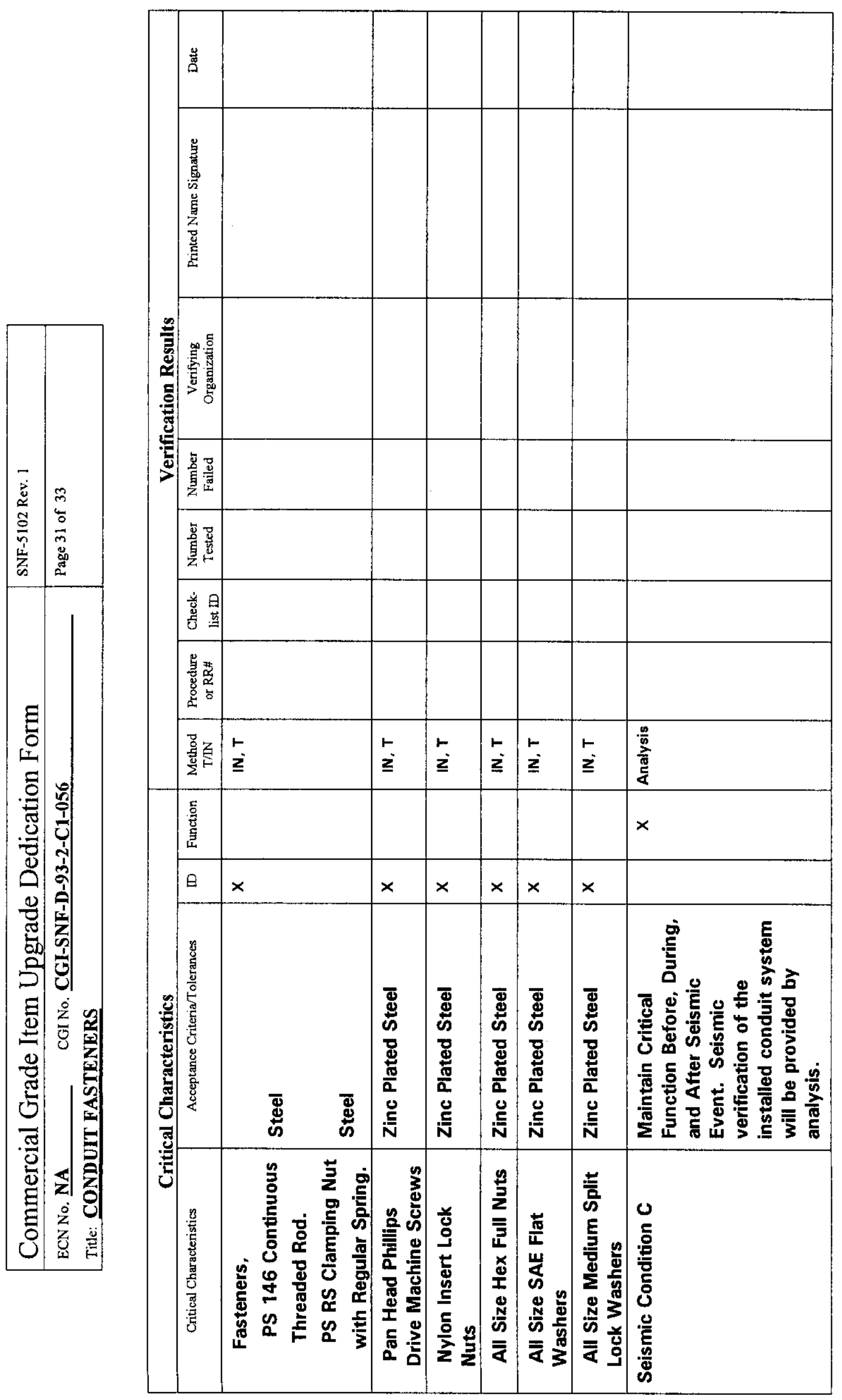




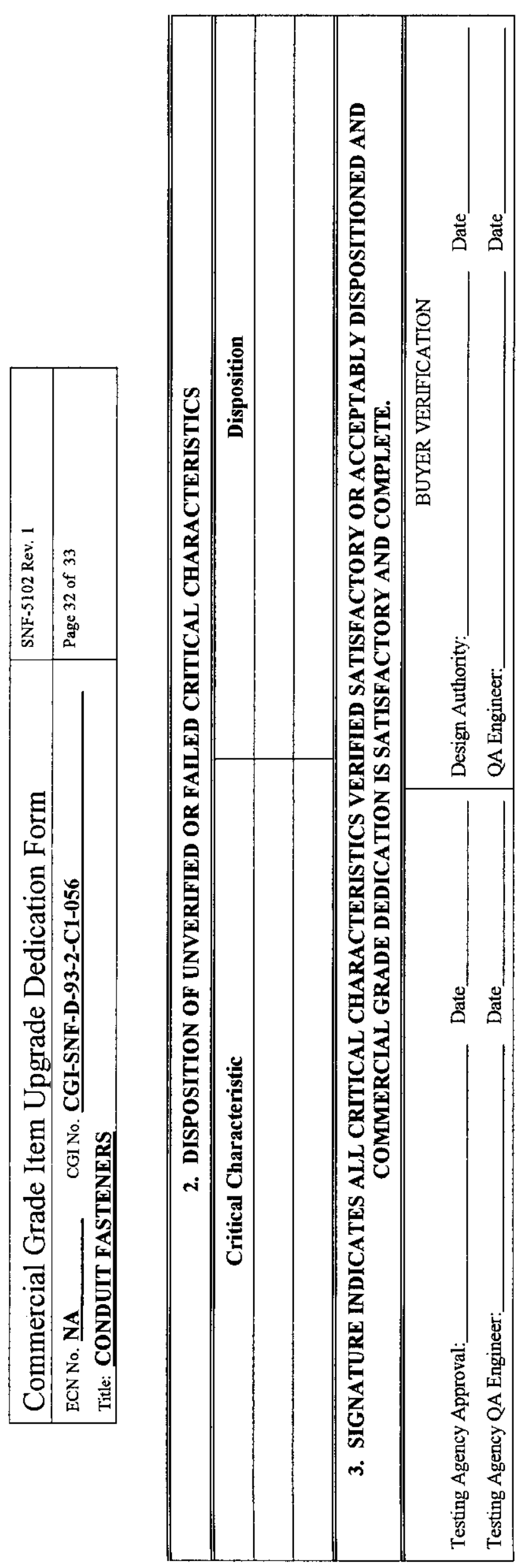




\begin{tabular}{|l|l|}
\hline Commercial Grade Item Upgrade Dedication Form & SNF-5102 Rev. 1 \\
\hline ECN No. NA CGI No. CGI-SNF-D-93-2-C1-056 & Page 33 of 33 \\
Title: CONDUIT FASTENERS & \\
\hline
\end{tabular}

\begin{tabular}{|c|c|c|}
\hline \multicolumn{3}{|c|}{ SECTION 6 CONTACTS / PHONE NUMBERS } \\
\hline \multicolumn{2}{|l|}{ Name } & Phone \\
\hline \multicolumn{2}{|l|}{ Design Authority } & ) \\
\hline \multicolumn{2}{|l|}{ QA } & ) \\
\hline \multicolumn{2}{|l|}{ QC } & ) \\
\hline \multicolumn{2}{|l|}{ Cog - Engineer } & ) \\
\hline \multicolumn{2}{|l|}{ CGI Engineer } & ) \\
\hline \multicolumn{2}{|l|}{ Procurement Engineer } & ) \\
\hline \multicolumn{3}{|c|}{ Other } \\
\hline \multirow{2}{*}{\multicolumn{3}{|c|}{$\begin{array}{l}\text { SECIION/ SUPRORING DOCUMENIAIION ROR THIS CHECKLIST } \\
\begin{array}{l|l}\text { Initial Procurement Documents } & \text { For Critical Characteristics }\end{array}\end{array}$}} \\
\hline & & \\
\hline \multicolumn{3}{|l|}{ [ ] Drawings: } \\
\hline \multicolumn{3}{|l|}{ [ ] Manuals (specify type \& number): } \\
\hline \multicolumn{3}{|l|}{ [ ] Design Calculations } \\
\hline \multicolumn{3}{|l|}{ [ ] Installation Instructions } \\
\hline \multicolumn{3}{|l|}{ [ ] Operation Instructions } \\
\hline \multicolumn{3}{|l|}{ I / Calibration Instructions } \\
\hline \multicolumn{3}{|l|}{ I I Manufacturer's Recommended Spare Parts List } \\
\hline \multicolumn{3}{|l|}{ [ ] Other: } \\
\hline \multicolumn{3}{|l|}{ Procurement Documents } \\
\hline \multicolumn{3}{|l|}{ [ ] Certificate of Conformance/Compliance } \\
\hline \multicolumn{3}{|l|}{ [ ] Seismic Qualification Certificate } \\
\hline \multicolumn{3}{|l|}{ [ ] Environmental Qualification Certificate } \\
\hline \multicolumn{3}{|l|}{ [ ] Test Report (s): } \\
\hline \multicolumn{3}{|l|}{ [ ] Inspection Report (s): } \\
\hline \multicolumn{3}{|l|}{ I | CMTRs for ASME Pressure Retaining Materials } \\
\hline \multicolumn{3}{|l|}{ [ ] Valve Seat Leakage Report } \\
\hline \multicolumn{3}{|l|}{ [ ] Weld Records } \\
\hline \multicolumn{3}{|l|}{ [ ] Material Traceability Record } \\
\hline [ ] Other: & & \\
\hline
\end{tabular}




\section{DISTRIBUTION SHEET}

To

Distribution

Project TitleNork Order

$W-441$, SNE-5102
From

T. Nuxal1, SNE-CVD
Page 1 of 1

Date $9 / 15 / 99$

EDT No. N/A

ECN No. 654680

\section{Name}

C. Miska/D. Whitehurst

C. Van Katwijk

A. Artzer (CVD Library)

R. Ramsgate

J. Brehm

P. Beaudet

P. Morrell (AVS)

M. Evarts (AI)

D. Marburger

SNF startup

SNF Project Files

\begin{tabular}{|l|c|l|l|l|}
\hline MSIN & $\begin{array}{c}\text { Text } \\
\text { With All } \\
\text { Attach. }\end{array}$ & Text Only & $\begin{array}{c}\text { Attach./ } \\
\text { Appendix } \\
\text { Only }\end{array}$ & $\begin{array}{c}\text { EDT/ECN } \\
\text { Only }\end{array}$ \\
\hline R3-86 & $x$ & & & \\
\hline R3-47 & $x$ & & & \\
\hline R3-86 & $x$ & & & \\
\hline R3-86 & $x$ & & & \\
\hline R3-26 & $x$ & & & \\
\hline S8-07 & $x$ & & & \\
\hline G1-50 & $x$ & & & \\
\hline N1-29 & $x$ & & & \\
\hline R3-26 & $x$ & & & \\
\hline B2-64 & $x$ & & & \\
\hline R3-11 & $x$ & & & \\
\hline
\end{tabular}

